\title{
Finite Series Representation of the Inverse Mittag-Leffler Function
}

\author{
John W. Hanneken and B. N. Narahari Achar \\ Department of Physics, University of Memphis, Memphis, TN 38152, USA \\ Correspondence should be addressed to John W. Hanneken; jhannekn@memphis.edu
}

Received 11 December 2013; Accepted 5 February 2014; Published 25 March 2014

Academic Editor: J. A. Tenreiro Machado

Copyright ( 2014 J. W. Hanneken and B. N. N. Achar. This is an open access article distributed under the Creative Commons Attribution License, which permits unrestricted use, distribution, and reproduction in any medium, provided the original work is properly cited.

\begin{abstract}
The inverse Mittag-Leffler function $E_{\alpha, \beta}^{-1}(z)$ is valuable in determining the value of the argument of a Mittag-Leffler function given the value of the function and it is not an easy problem. A finite series representation of the inverse Mittag-Leffler function has been found for a range of the parameters $\alpha$ and $\beta$; specifically, $0<\alpha<1 / 2$ for $\beta=1$ and for $\beta=2$. This finite series representation of the inverse Mittag-Leffler function greatly expedites its evaluation and has been illustrated with a number of examples. This represents a significant advancement in the understanding of Mittag-Leffler functions.
\end{abstract}

\section{Introduction}

The Mittag-Leffler function $E_{\alpha, \beta}(z)$ is defined by the power series [1]

$$
E_{\alpha, \beta}(z)=\sum_{k=0}^{\infty} \frac{z^{k}}{\Gamma(\alpha k+\beta)} \quad z \in \mathbb{C}
$$

While the argument $z$ and the parameters $\alpha$ and $\beta$ can in general be complex provided $\operatorname{Re} \alpha>0$, in this work $z, \alpha$, and $\beta$ will be restricted to those values most commonly found in physical problems; namely, the argument $z$ will be restricted to real numbers and $\alpha$ and $\beta$ will be restricted to positive real numbers. The Mittag-Leffler function is a generalization of the exponential function and arises frequently in the solutions of differential and/or integral equations of fractional (noninteger) order in much the same way as the exponential function appears in solutions of differential equations of integer order. Thus, Mittag-Leffler functions play a fundamental role in the theory of fractional differential equations. Consequently, books devoted to the subject of fractional differential equations (i.e., Podlubny [2], Kilbas et al. [3], and Diethelm [4]) all contain sections on the Mittag-Leffler functions. In addition to their inherent mathematical interest, Mittag-Leffler functions are also important in theoretical and applied physics and all the sciences (i.e., Hilfer [5], Mainardi [6], and Magin [7]). The works of Mainardi and Gorenflo [8], Magin [9], Berberan-Santos [10], Gupta and Debnath [11], and Haubold et al. [12] are a few of the numerous articles also worth noting.

The inverse Mittag-Leffler function $E_{\alpha, \beta}^{-1}(z)$ is defined as the solution of (2) [13]

$$
E_{\alpha, \beta}^{-1}\left[E_{\alpha, \beta}(z)\right]=z .
$$

Despite the inherent importance of Mittag-Leffler functions in fractional differential equations, with the wealth of analytical information about $E_{\alpha, \beta}(z)$, the inverse $E_{\alpha, \beta}^{-1}(z)$ has been largely unexplored. The one exception is the excellent work of Hilfer and Seybold [13] who have determined its principal branch numerically.

The power series representation of any Mittag-Leffler function can be inverted yielding an infinite series for the inverse. However, these infinite series are slow to converge and terminating the series always introduces error which is hard to evaluate. This present work identifies regions in the domain of $\alpha$ and $\beta$ where the inverse of the Mittag-Leffler function can be written as a finite series. This represents the first time the inverse Mittag-Leffler function has been written as a finite series as opposed to an infinite series which greatly 
expedites its evaluation. Before deriving these expressions for the inverse Mittag-Leffler function, a brief review of the theory of power series and their inverses is in order.

\section{Theory}

Consider the convergent series which expresses the function $w=f(z)$ in terms of powers of $\left(z-z_{o}\right)$ with the corresponding coefficients $a_{k}$ given by

$$
\begin{aligned}
w & =f(z)=\sum_{k=0}^{\infty} a_{k}\left(z-z_{o}\right)^{k} \\
& =a_{o}+a_{1}\left(z-z_{o}\right)+a_{2}\left(z-z_{o}\right)^{2}+\cdots
\end{aligned}
$$

The inversion of the function $f(z)$ requires only the sole assumption that $a_{1} \neq 0$. That is, there exists one and only one function which represents the inverse of the $f(z), z-z_{o}=$ $f^{-1}(w)$, which is expressible by a convergent power series of the form [14]

$$
\begin{aligned}
z-z_{o} & =f^{-1}(w)=\sum_{k=1}^{\infty} b_{k}\left(w-a_{o}\right)^{k} \\
& =b_{1}\left(w-a_{o}\right)+b_{2}\left(w-a_{o}\right)^{2}+\cdots
\end{aligned}
$$

The process of finding the series expansion for $f^{-1}(w)$ is called reversion of the series. The coefficients $b_{k}$ can be determined in terms of the coefficients $a_{k}$ by substituting (3) into (4) and equating coefficients of like powers of $\left(z-z_{o}\right)^{k}$ on both sides of the equation yielding

$$
\begin{array}{ll}
b_{1}=\frac{1}{a_{1}}, & b_{3}=\frac{1}{a_{1}^{5}}\left(2 a_{2}^{2}-a_{1} a_{3}\right), \\
b_{2}=-\frac{a_{2}}{a_{1}^{3}}, & b_{4}=\frac{1}{a_{1}^{7}}\left(5 a_{1} a_{2} a_{3}-a_{1}^{2} a_{4}-5 a_{2}^{3}\right) .
\end{array}
$$

The coefficients $b_{1}, b_{2}, b_{3}, \ldots, b_{7}$ can be found in the literature [15-17]. An explicit expression for the coefficients $b_{k}$ can be derived using the Lagrange inversion theorem. If $f(z)$ is analytic at $z=z_{o}$ and $f^{\prime}\left(z_{o}\right) \neq 0$, then the inverse of $f(z)$ exists and is analytic about $f\left(z_{o}\right)$. Furthermore, if $f(z)=$ $w$, the Lagrange inversion theorem gives the Taylor series expansion of the inverse function $f^{-1}(w)$ as [15]

$$
f^{-1}(w)=z-z_{o}=\sum_{k=1}^{\infty} \frac{\left(w-a_{o}\right)^{k}}{k !} \frac{d^{k-1}}{d z^{k-1}}\left\{\frac{\left(z-z_{o}\right)^{k}}{\left[f(z)-a_{o}\right]^{k}}\right\}_{z=z_{o}} .
$$

The coefficients $b_{k}$ are determined by comparing (6) and (4) yielding

$$
b_{k}=\frac{1}{k !} \frac{d^{k-1}}{d z^{k-1}}\left\{\frac{\left(z-z_{o}\right)^{k}}{\left[f(z)-a_{o}\right]^{k}}\right\}_{z=z_{o}} .
$$

Substituting $f(z)-a_{o}$ from (3) yields

$$
b_{k}=\frac{1}{k !} \frac{d^{k-1}}{d z^{k-1}}\left\{\left[a_{1}+a_{2}\left(z-z_{o}\right)+a_{3}\left(z-z_{o}\right)^{2}+\cdots\right]^{-k}\right\}_{z=z_{o}} .
$$

Factoring out $a_{1}^{k}$ in (8) and defining $x=z-z_{o}$ yields

$$
\begin{aligned}
b_{k}= & \frac{1}{a_{1}^{k} k !} \frac{d^{k-1}}{d z^{k-1}} \\
& \times\left\{\left[1+\left(\frac{a_{2}}{a_{1}}\right) x+\left(\frac{a_{3}}{a_{1}}\right) x^{2}+\left(\frac{a_{4}}{a_{1}}\right) x^{3}+\cdots\right]^{-k}\right\}_{x=0} .
\end{aligned}
$$

Using the multinomial expansion and performing the required differentiation yields the desired result [18]

$$
\begin{aligned}
b_{k}= & \frac{1}{k a_{1}^{k}} \\
& \times \sum_{s, t, u, \ldots}(-1)^{s+t+u+\cdots} \frac{(k)(k+1) \cdots(k-1+s+t+u \cdots)}{s ! t ! u ! \cdots} \\
& \times\left(\frac{a_{2}}{a_{1}}\right)^{s}\left(\frac{a_{3}}{a_{1}}\right)^{t}\left(\frac{a_{3}}{a_{1}}\right)^{u} \cdots,
\end{aligned}
$$

where $s+2 t+3 u+\cdots=k-1$ and the numbers $s, t, u, \ldots$ are nonnegative integers and the summation extends over all partitions of $k-1$. For example, $b_{5}$ contains 5 terms since the Diophantine equation $s+2 t+3 u+4 v=4$ has 5 integer solutions or partitions. The number of partitions for $k=11$ is 42; for $k=51$ there are 204226 partitions and for $k=101$ the number of partitions is 190569292 . Consequently, the explicit tabulation of the full expression for the coefficients $b_{k}$ rapidly becomes a rather tedious task. Nevertheless, the coefficients $b_{1}, b_{2}, b_{3}, \ldots, b_{14}$ are given in Table 1 . An equivalent expression for the general term $b_{k}$ in the reversion of series is given in a different form by McMahon [19].

By an appropriate change of variables it is always possible to write the power series in a form which results in simplified expressions for the coefficients in the reversed power series. Equation (3) can be rewritten as

$$
\frac{w-a_{o}}{a_{1}}=\left(z-z_{o}\right)\left[1+\frac{a_{2}}{a_{1}}\left(z-z_{o}\right)+\frac{a_{3}}{a_{1}}\left(z-z_{o}\right)^{2}+\cdots\right] .
$$

Defining the new variables $W=\left(w-a_{o}\right) / a_{1}, A_{1}=-a_{2} / a_{1}$, $A_{2}=-a_{3} / a_{1}$, and so forth, (11) becomes

$$
W=\left(z-z_{o}\right)\left[1-\sum_{k=1}^{\infty} A_{k}\left(z-z_{o}\right)^{k}\right]
$$

and the reversed series is given by

$$
\left(z-z_{o}\right)=W\left[1-\sum_{k=1}^{\infty} B_{k} W^{k}\right]
$$


TABLE 1: Coefficients of the inverse function for a power series.

\begin{tabular}{|c|c|}
\hline$k$ & Coefficient $b_{k}$ \\
\hline 1 & 1 \\
\hline & $\overline{a_{1}}$ \\
\hline 2 & $-\frac{a_{2}}{a_{1}^{3}}$ \\
\hline 3 & $\frac{1}{a_{1}^{5}}\left(2 a_{2}^{2}-a_{1} a_{3}\right)$ \\
\hline 4 & $\frac{1}{a_{1}^{7}}\left(-5 a_{2}^{3}+5 a_{1} a_{2} a_{3}-a_{1}^{2} a_{4}\right)$ \\
\hline 5 & $\frac{1}{a_{1}^{9}}\left(14 a_{2}^{4}-21 a_{1} a_{2}^{2} a_{3}+3 a_{1}^{2} a_{3}^{2}+6 a_{1}^{2} a_{2} a_{4}-a_{1}^{3} a_{5}\right)$ \\
\hline 6 & $\frac{1}{a_{1}^{11}}\left(-42 a_{2}^{5}+84 a_{1} a_{2}^{3} a_{3}-28 a_{1}^{2} a_{2} a_{3}^{2}-28 a_{1}^{2} a_{2}^{2} a_{4}+7 a_{1}^{3} a_{3} a_{4}+7 a_{1}^{3} a_{2} a_{5}-a_{1}^{4} a_{6}\right)$ \\
\hline 7 & $\frac{1}{a_{1}^{13}}\left(132 a_{2}^{6}-330 a_{1} a_{2}^{4} a_{3}+180 a_{1}^{2} a_{2}^{2} a_{3}^{2}-12 a_{1}^{3} a_{3}^{3}+120 a_{1}^{2} a_{2}^{3} a_{4}-72 a_{1}^{3} a_{2} a_{3} a_{4}+4 a_{1}^{4} a_{4}^{2}-36 a_{1}^{3} a_{2}^{2} a_{5}+8 a_{1}^{4} a_{3} a_{5}+8 a_{1}^{4} a_{2} a_{6}-a_{1}^{5} a_{7}\right)$ \\
\hline 8 & $\begin{array}{l}\frac{1}{a_{1}^{15}}\left(-429 a_{2}^{7}+1287 a_{1} a_{2}^{5} a_{3}-990 a_{1}^{2} a_{2}^{3} a_{3}^{2}+165 a_{1}^{3} a_{2} a_{3}^{3}-495 a_{1}^{2} a_{2}^{4} a_{4}+495 a_{1}^{3} a_{2}^{2} a_{3} a_{4}-45 a_{1}^{4} a_{3}^{2} a_{4}-45 a_{1}^{4} a_{2} a_{4}^{2}+165 a_{1}^{3} a_{2}^{3} a_{5}\right. \\
\left.\quad-90 a_{1}^{4} a_{2} a_{3} a_{5}+9 a_{1}^{5} a_{4} a_{5}-45 a_{1}^{4} a_{2}^{2} a_{6}+9 a_{1}^{5} a_{3} a_{6}+9 a_{1}^{5} a_{2} a_{7}-a_{1}^{6} a_{8}\right)\end{array}$ \\
\hline 9 & $\begin{array}{l}\frac{1}{a_{1}^{17}}\left(1430 a_{2}^{8}-5005 a_{1} a_{2}^{6} a_{3}+5005 a_{1}^{2} a_{2}^{4} a_{3}^{2}-1430 a_{1}^{3} a_{2}^{2} a_{3}^{3}+55 a_{1}^{4} a_{3}^{4}+2002 a_{1}^{2} a_{2}^{5} a_{4}-2860 a_{1}^{3} a_{2}^{3} a_{3} a_{4}+660 a_{1}^{4} a_{2} a_{3}^{2} a_{4}+330 a_{1}^{4} a_{2}^{2} a_{4}^{2}\right. \\
\quad-55 a_{1}^{5} a_{3} a_{4}^{2}-715 a_{1}^{3} a_{2}^{4} a_{5}+660 a_{1}^{4} a_{2}^{2} a_{3} a_{5}-55 a_{1}^{5} a_{3}^{2} a_{5}-110 a_{1}^{5} a_{2} a_{4} a_{5}+5 a_{1}^{6} a_{5}^{2}+220 a_{1}^{4} a_{2}^{3} a_{6}-110 a_{1}^{5} a_{2} a_{3} a_{6}+10 a_{1}^{6} a_{4} a_{6} \\
\left.\quad-55 a_{1}^{5} a_{2}^{2} a_{7}+10 a_{1}^{6} a_{3} a_{7}+10 a_{1}^{6} a_{2} a_{8}-a_{1}^{7} a_{9}\right)\end{array}$ \\
\hline 10 & $\begin{array}{l}\frac{1}{a_{1}^{19}}\left(-4862 a_{2}^{9}+19448 a_{1} a_{2}^{7} a_{3}-24024 a_{1}^{2} a_{2}^{5} a_{3}^{2}+10010 a_{1}^{3} a_{2}^{3} a_{3}^{3}-1001 a_{1}^{4} a_{2} a_{3}^{4}-8008 a_{1}^{2} a_{2}^{6} a_{4}+15015 a_{1}^{3} a_{2}^{4} a_{3} a_{4}-6006 a_{1}^{4} a_{2}^{2} a_{3}^{2} a_{4}\right. \\
+286 a_{1}^{5} a_{3}^{3} a_{4}-2002 a_{1}^{4} a_{2}^{3} a_{4}^{2}+858 a_{1}^{5} a_{2} a_{3} a_{4}^{2}-22 a_{1}^{6} a_{4}^{3}+3003 a_{1}^{3} a_{2}^{5} a_{5}-4004 a_{1}^{4} a_{2}^{3} a_{3} a_{5}+858 a_{1}^{5} a_{2} a_{3}^{2} a_{5}+858 a_{1}^{5} a_{2}^{2} a_{4} a_{5} \\
\quad-132 a_{1}^{6} a_{3} a_{4} a_{5}-66 a_{1}^{6} a_{2} a_{5}^{2}-1001 a_{1}^{4} a_{2}^{4} a_{6}+858 a_{1}^{5} a_{2}^{2} a_{3} a_{6}-66 a_{1}^{6} a_{3}^{2} a_{6}-132 a_{1}^{6} a_{2} a_{4} a_{6}+11 a_{1}^{7} a_{5} a_{6}+286 a_{1}^{5} a_{2}^{3} a_{7} \\
\left.-132 a_{1}^{6} a_{2} a_{3} a_{7}+11 a_{1}^{7} a_{4} a_{7}-66 a_{1}^{6} a_{2}^{2} a_{8}+11 a_{1}^{7} a_{3} a_{8}+11 a_{1}^{7} a_{2} a_{9}-a_{1}^{8} a_{10}\right)\end{array}$ \\
\hline 11 & $\begin{array}{l}\frac{1}{a_{1}^{21}}\left(16796 a_{2}^{10}-75582 a_{1} a_{2}^{8} a_{3}+111384 a_{1}^{2} a_{2}^{6} a_{3}^{2}-61880 a_{1}^{3} a_{2}^{4} a_{3}^{3}+10920 a_{1}^{4} a_{2}^{2} a_{3}^{4}-273 a_{1}^{5} a_{3}^{5}+31824 a_{1}^{2} a_{2}^{7} a_{4}-74256 a_{1}^{3} a_{2}^{5} a_{3} a_{4}\right. \\
+43680 a_{1}^{4} a_{2}^{3} a_{3}^{2} a_{4}-5460 a_{1}^{5} a_{2} a_{3}^{3} a_{4}+10920 a_{1}^{4} a_{2}^{4} a_{4}^{2}+-8190 a_{1}^{5} a_{2}^{2} a_{3} a_{4}^{2}+546 a_{1}^{6} a_{3}^{2} a_{4}^{2}+364 a_{1}^{6} a_{2} a_{4}^{3}-12376 a_{1}^{3} a_{2}^{6} a_{5} \\
+21840 a_{1}^{4} a_{2}^{4} a_{3} a_{5}+-8190 a_{1}^{5} a_{2}^{2} a_{3}^{2} a_{5}+364 a_{1}^{6} a_{3}^{3} a_{5}-5460 a_{1}^{5} a_{2}^{3} a_{4} a_{5}+2184 a_{1}^{6} a_{2} a_{3} a_{4} a_{5}-78 a_{1}^{7} a_{4}^{2} a_{5}+546 a_{1}^{6} a_{2}^{2} a_{5}^{2} \\
\quad-78 a_{1}^{7} a_{3} a_{5}^{2}+4368 a_{1}^{4} a_{2}^{5} a_{6}-5460 a_{1}^{5} a_{2}^{3} a_{3} a_{6}+1092 a_{1}^{6} a_{2} a_{3}^{2} a_{6}+1092 a_{1}^{6} a_{2}^{2} a_{4} a_{6}-156 a_{1}^{7} a_{3} a_{4} a_{6}-156 a_{1}^{7} a_{2} a_{5} a_{6}+6 a_{1}^{8} a_{6}^{2} \\
\quad-1365 a_{1}^{5} a_{2}^{4} a_{7}+1092 a_{1}^{6} a_{2}^{2} a_{3} a_{7}+-78 a_{1}^{7} a_{3}^{2} a_{7}-156 a_{1}^{7} a_{2} a_{4} a_{7}+12 a_{1}^{8} a_{5} a_{7}+364 a_{1}^{6} a_{2}^{3} a_{8}-156 a_{1}^{7} a_{2} a_{3} a_{8}+12 a_{1}^{8} a_{4} a_{8} \\
\left.+-78 a_{1}^{7} a_{2}^{2} a_{9}+12 a_{1}^{8} a_{3} a_{9}+12 a_{1}^{8} a_{2} a_{10}-a_{1}^{9} a_{11}\right)\end{array}$ \\
\hline 12 & $\begin{array}{l}\frac{1}{a_{1}^{23}}\left(-58786 a_{2}^{11}+293930 a_{1} a_{2}^{9} a_{3}-503880 a_{1}^{2} a_{2}^{7} a_{3}^{2}+352716 a_{1}^{3} a_{2}^{5} a_{3}^{3}-92820 a_{1}^{4} a_{2}^{3} a_{3}^{4}+6188 a_{1}^{5} a_{2} a_{3}^{5}+-125970 a_{1}^{2} a_{2}^{8} a_{4}\right. \\
\quad+352716 a_{1}^{3} a_{2}^{6} a_{3} a_{4}-278460 a_{1}^{4} a_{2}^{4} a_{3}^{2} a_{4}+61880 a_{1}^{5} a_{2}^{2} a_{3}^{3} a_{4}-1820 a_{1}^{6} a_{3}^{4} a_{4}+-55692 a_{1}^{4} a_{2}^{5} a_{4}^{2}+61880 a_{1}^{5} a_{2}^{3} a_{3} a_{4}^{2} \\
\quad-10920 a_{1}^{6} a_{2} a_{3}^{2} a_{4}^{2}-3640 a_{1}^{6} a_{2}^{2} a_{4}^{3}+455 a_{1}^{7} a_{3} a_{4}^{3}+50388 a_{1}^{3} a_{2}^{7} a_{5}-111384 a_{1}^{4} a_{2}^{5} a_{3} a_{5}+61880 a_{1}^{5} a_{2}^{3} a_{3}^{2} a_{5}-7280 a_{1}^{6} a_{2} a_{3}^{3} a_{5} \\
\quad+30940 a_{1}^{5} a_{2}^{4} a_{4} a_{5}+-21840 a_{1}^{6} a_{2}^{2} a_{3} a_{4} a_{5}+1365 a_{1}^{7} a_{3}^{2} a_{4} a_{5}+1365 a_{1}^{7} a_{2} a_{4}^{2} a_{5}-3640 a_{1}^{6} a_{2}^{3} a_{5}^{2}+1365 a_{1}^{7} a_{2} a_{3} a_{5}^{2}+-91 a_{1}^{8} a_{4} a_{5}^{2} \\
\quad-18564 a_{1}^{4} a_{2}^{6} a_{6}+30940 a_{1}^{5} a_{2}^{4} a_{3} a_{6}-10920 a_{1}^{6} a_{2}^{2} a_{3}^{2} a_{6}+455 a_{1}^{7} a_{3}^{3} a_{6}+-7280 a_{1}^{6} a_{2}^{3} a_{4} a_{6}+2730 a_{1}^{7} a_{2} a_{3} a_{4} a_{6}-91 a_{1}^{8} a_{4}^{2} a_{6} \\
\quad+1365 a_{1}^{7} a_{2}^{2} a_{5} a_{6}-182 a_{1}^{8} a_{3} a_{5} a_{6}+-91 a_{1}^{8} a_{2} a_{6}^{2}+6188 a_{1}^{5} a_{2}^{5} a_{7}-7280 a_{1}^{6} a_{2}^{3} a_{3} a_{7}+1365 a_{1}^{7} a_{2} a_{3}^{2} a_{7}+1365 a_{1}^{7} a_{2}^{2} a_{4} a_{7} \\
\quad+-182 a_{1}^{8} a_{3} a_{4} a_{7}-182 a_{1}^{8} a_{2} a_{5} a_{7}+13 a_{1}^{9} a_{6} a_{7}-1820 a_{1}^{6} a_{2}^{4} a_{8}+1365 a_{1}^{7} a_{2}^{2} a_{3} a_{8}-91 a_{1}^{8} a_{3}^{2} a_{8}+-182 a_{1}^{8} a_{2} a_{4} a_{8}+13 a_{1}^{9} a_{5} a_{8} \\
\left.+455 a_{1}^{7} a_{2}^{3} a_{9}-182 a_{1}^{8} a_{2} a_{3} a_{9}+13 a_{1}^{9} a_{4} a_{9}-91 a_{1}^{8} a_{2}^{2} a_{10}+13 a_{1}^{9} a_{3} a_{10}+13 a_{1}^{9} a_{2} a_{11}-a_{1}^{10} a_{12}\right)\end{array}$ \\
\hline
\end{tabular}


TABle 1: Continued.

$k$

Coefficient $b_{k}$

$\frac{1}{a_{1}^{25}}\left(208012 a_{2}^{12}-1144066 a_{1} a_{2}^{10} a_{3}+2238390 a_{1}^{2} a_{2}^{8} a_{3}^{2}-1899240 a_{1}^{3} a_{2}^{6} a_{3}^{3}+678300 a_{1}^{4} a_{2}^{4} a_{3}^{4}+-81396 a_{1}^{5} a_{2}^{2} a_{3}^{5}+1428 a_{1}^{6} a_{3}^{6}\right.$

$+497420 a_{1}^{2} a_{2}^{9} a_{4}-1627920 a_{1}^{3} a_{2}^{7} a_{3} a_{4}+1627920 a_{1}^{4} a_{2}^{5} a_{3}^{2} a_{4}+-542640 a_{1}^{5} a_{2}^{3} a_{3}^{3} a_{4}+42840 a_{1}^{6} a_{2} a_{3}^{4} a_{4}+271320 a_{1}^{4} a_{2}^{6} a_{4}^{2}$

$-406980 a_{1}^{5} a_{2}^{4} a_{3} a_{4}^{2}+128520 a_{1}^{6} a_{2}^{2} a_{3}^{2} a_{4}^{2}-4760 a_{1}^{7} a_{3}^{3} a_{4}^{2}+28560 a_{1}^{6} a_{2}^{3} a_{4}^{3}-9520 a_{1}^{7} a_{2} a_{3} a_{4}^{3}+140 a_{1}^{8} a_{4}^{4}+-203490 a_{1}^{3} a_{2}^{8} a_{5}$

$+542640 a_{1}^{4} a_{2}^{6} a_{3} a_{5}-406980 a_{1}^{5} a_{2}^{4} a_{3}^{2} a_{5}+85680 a_{1}^{6} a_{2}^{2} a_{3}^{3} a_{5}-2380 a_{1}^{7} a_{3}^{4} a_{5}+-162792 a_{1}^{5} a_{2}^{5} a_{4} a_{5}+171360 a_{1}^{6} a_{2}^{3} a_{3} a_{4} a_{5}$

$-28560 a_{1}^{7} a_{2} a_{3}^{2} a_{4} a_{5}-14280 a_{1}^{7} a_{2}^{2} a_{4}^{2} a_{5}+1680 a_{1}^{8} a_{3} a_{4}^{2} a_{5}+21420 a_{1}^{6} a_{2}^{4} a_{5}^{2}-14280 a_{1}^{7} a_{2}^{2} a_{3} a_{5}^{2}+840 a_{1}^{8} a_{3}^{2} a_{5}^{2}+1680 a_{1}^{8} a_{2} a_{4} a_{5}^{2}$

13

$-35 a_{1}^{9} a_{5}^{3}+77520 a_{1}^{4} a_{2}^{7} a_{6}-162792 a_{1}^{5} a_{2}^{5} a_{3} a_{6}+85680 a_{1}^{6} a_{2}^{3} a_{3}^{2} a_{6}-9520 a_{1}^{7} a_{2} a_{3}^{3} a_{6}+42840 a_{1}^{6} a_{2}^{4} a_{4} a_{6}+-28560 a_{1}^{7} a_{2}^{2} a_{3} a_{4} a_{6}$

$+1680 a_{1}^{8} a_{3}^{2} a_{4} a_{6}+1680 a_{1}^{8} a_{2} a_{4}^{2} a_{6}-9520 a_{1}^{7} a_{2}^{3} a_{5} a_{6}+3360 a_{1}^{8} a_{2} a_{3} a_{5} a_{6}+-210 a_{1}^{9} a_{4} a_{5} a_{6}+840 a_{1}^{8} a_{2}^{2} a_{6}^{2}-105 a_{1}^{9} a_{3} a_{6}^{2}$

$-27132 a_{1}^{5} a_{2}^{6} a_{7}+42840 a_{1}^{6} a_{2}^{4} a_{3} a_{7}-14280 a_{1}^{7} a_{2}^{2} a_{3}^{2} a_{7}+560 a_{1}^{8} a_{3}^{3} a_{7}-9520 a_{1}^{7} a_{2}^{3} a_{4} a_{7}+3360 a_{1}^{8} a_{2} a_{3} a_{4} a_{7}-105 a_{1}^{9} a_{4}^{2} a_{7}$

$+1680 a_{1}^{8} a_{2}^{2} a_{5} a_{7}-210 a_{1}^{9} a_{3} a_{5} a_{7}+-210 a_{1}^{9} a_{2} a_{6} a_{7}+7 a_{1}^{10} a_{7}^{2}+8568 a_{1}^{6} a_{2}^{5} a_{8}-9520 a_{1}^{7} a_{2}^{3} a_{3} a_{8}+1680 a_{1}^{8} a_{2} a_{3}^{2} a_{8}+1680 a_{1}^{8} a_{2}^{2} a_{4} a_{8}$

$+-210 a_{1}^{9} a_{3} a_{4} a_{8}-210 a_{1}^{9} a_{2} a_{5} a_{8}+14 a_{1}^{10} a_{6} a_{8}-2380 a_{1}^{7} a_{2}^{4} a_{9}+1680 a_{1}^{8} a_{2}^{2} a_{3} a_{9}-105 a_{1}^{9} a_{3}^{2} a_{9}+-210 a_{1}^{9} a_{2} a_{4} a_{9}+14 a_{1}^{10} a_{5} a_{9}$

$\left.+560 a_{1}^{8} a_{2}^{3} a_{10}-210 a_{1}^{9} a_{2} a_{3} a_{10}+14 a_{1}^{10} a_{4} a_{10}-105 a_{1}^{9} a_{2}^{2} a_{11}+14 a_{1}^{10} a_{3} a_{11}+14 a_{1}^{10} a_{2} a_{12}-a_{1}^{11} a_{13}\right)$

$\frac{1}{a_{1}^{27}}\left(-742900 a_{2}^{13}+4457400 a_{1} a_{2}^{11} a_{3}-9806280 a_{1}^{2} a_{2}^{9} a_{3}^{2}+9806280 a_{1}^{3} a_{2}^{7} a_{3}^{3}-4476780 a_{1}^{4} a_{2}^{5} a_{3}^{4}+813960 a_{1}^{5} a_{2}^{3} a_{3}^{5}-38760 a_{1}^{6} a_{2} a_{3}^{6}\right.$

$-1961256 a_{1}^{2} a_{2}^{10} a_{4}+7354710 a_{1}^{3} a_{2}^{8} a_{3} a_{4}-8953560 a_{1}^{4} a_{2}^{6} a_{3}^{2} a_{4}+4069800 a_{1}^{5} a_{2}^{4} a_{3}^{3} a_{4}-581400 a_{1}^{6} a_{2}^{2} a_{3}^{4} a_{4}+11628 a_{1}^{7} a_{3}^{5} a_{4}$

$-1279080 a_{1}^{4} a_{2}^{7} a_{4}^{2}+2441880 a_{1}^{5} a_{2}^{5} a_{3} a_{4}^{2}-1162800 a_{1}^{6} a_{2}^{3} a_{3}^{2} a_{4}^{2}+116280 a_{1}^{7} a_{2} a_{3}^{3} a_{4}^{2}-193800 a_{1}^{6} a_{2}^{4} a_{4}^{3}+116280 a_{1}^{7} a_{2}^{2} a_{3} a_{4}^{3}$

$+-6120 a_{1}^{8} a_{3}^{2} a_{4}^{3}-3060 a_{1}^{8} a_{2} a_{4}^{4}+817190 a_{1}^{3} a_{2}^{9} a_{5}-2558160 a_{1}^{4} a_{2}^{7} a_{3} a_{5}+2441880 a_{1}^{5} a_{2}^{5} a_{3}^{2} a_{5}+-775200 a_{1}^{6} a_{2}^{3} a_{3}^{3} a_{5}$

$+58140 a_{1}^{7} a_{2} a_{3}^{4} a_{5}+813960 a_{1}^{5} a_{2}^{6} a_{4} a_{5}-1162800 a_{1}^{6} a_{2}^{4} a_{3} a_{4} a_{5}+348840 a_{1}^{7} a_{2}^{2} a_{3}^{2} a_{4} a_{5}-12240 a_{1}^{8} a_{3}^{3} a_{4} a_{5}+116280 a_{1}^{7} a_{2}^{3} a_{4}^{2} a_{5}$

$-36720 a_{1}^{8} a_{2} a_{3} a_{4}^{2} a_{5}+680 a_{1}^{9} a_{4}^{3} a_{5}-116280 a_{1}^{6} a_{2}^{5} a_{5}^{2}+116280 a_{1}^{7} a_{2}^{3} a_{3} a_{5}^{2}-18360 a_{1}^{8} a_{2} a_{3}^{2} a_{5}^{2}-18360 a_{1}^{8} a_{2}^{2} a_{4} a_{5}^{2}+2040 a_{1}^{9} a_{3} a_{4} a_{5}^{2}$

$+680 a_{1}^{9} a_{2} a_{5}^{3}-319770 a_{1}^{4} a_{2}^{8} a_{6}+813960 a_{1}^{5} a_{2}^{6} a_{3} a_{6}-581400 a_{1}^{6} a_{2}^{4} a_{3}^{2} a_{6}+116280 a_{1}^{7} a_{2}^{2} a_{3}^{3} a_{6}-3060 a_{1}^{8} a_{3}^{4} a_{6}-232560 a_{1}^{6} a_{2}^{5} a_{4} a_{6}$

14

$+232560 a_{1}^{7} a_{2}^{3} a_{3} a_{4} a_{6}+-36720 a_{1}^{8} a_{2} a_{3}^{2} a_{4} a_{6}-18360 a_{1}^{8} a_{2}^{2} a_{4}^{2} a_{6}+2040 a_{1}^{9} a_{3} a_{4}^{2} a_{6}+58140 a_{1}^{7} a_{2}^{4} a_{5} a_{6}-36720 a_{1}^{8} a_{2}^{2} a_{3} a_{5} a_{6}$

$+2040 a_{1}^{9} a_{3}^{2} a_{5} a_{6}+4080 a_{1}^{9} a_{2} a_{4} a_{5} a_{6}-120 a_{1}^{10} a_{5}^{2} a_{6}-6120 a_{1}^{8} a_{2}^{3} a_{6}^{2}+2040 a_{1}^{9} a_{2} a_{3} a_{6}^{2}-120 a_{1}^{10} a_{4} a_{6}^{2}+116280 a_{1}^{5} a_{2}^{7} a_{7}$

$-232560 a_{1}^{6} a_{2}^{5} a_{3} a_{7}+116280 a_{1}^{7} a_{2}^{3} a_{3}^{2} a_{7}-12240 a_{1}^{8} a_{2} a_{3}^{3} a_{7}+58140 a_{1}^{7} a_{2}^{4} a_{4} a_{7}+-36720 a_{1}^{8} a_{2}^{2} a_{3} a_{4} a_{7}+2040 a_{1}^{9} a_{3}^{2} a_{4} a_{7}$

$+2040 a_{1}^{9} a_{2} a_{4}^{2} a_{7}-12240 a_{1}^{8} a_{2}^{3} a_{5} a_{7}+4080 a_{1}^{9} a_{2} a_{3} a_{5} a_{7}+-240 a_{1}^{10} a_{4} a_{5} a_{7}+2040 a_{1}^{9} a_{2}^{2} a_{6} a_{7}-240 a_{1}^{10} a_{3} a_{6} a_{7}-120 a_{1}^{10} a_{2} a_{7}^{2}$

$-38760 a_{1}^{6} a_{2}^{6} a_{8}+58140 a_{1}^{7} a_{2}^{4} a_{3} a_{8}-18360 a_{1}^{8} a_{2}^{2} a_{3}^{2} a_{8}+680 a_{1}^{9} a_{3}^{3} a_{8}-12240 a_{1}^{8} a_{2}^{3} a_{4} a_{8}+4080 a_{1}^{9} a_{2} a_{3} a_{4} a_{8}+-120 a_{1}^{10} a_{4}^{2} a_{8}$

$+2040 a_{1}^{9} a_{2}^{2} a_{5} a_{8}-240 a_{1}^{10} a_{3} a_{5} a_{8}-240 a_{1}^{10} a_{2} a_{6} a_{8}+15 a_{1}^{11} a_{7} a_{8}+11628 a_{1}^{7} a_{2}^{5} a_{9}+-12240 a_{1}^{8} a_{2}^{3} a_{3} a_{9}+2040 a_{1}^{9} a_{2} a_{3}^{2} a_{9}$

$+2040 a_{1}^{9} a_{2}^{2} a_{4} a_{9}-240 a_{1}^{10} a_{3} a_{4} a_{9}-240 a_{1}^{10} a_{2} a_{5} a_{9}+15 a_{1}^{11} a_{6} a_{9}-3060 a_{1}^{8} a_{2}^{4} a_{10}+2040 a_{1}^{9} a_{2}^{2} a_{3} a_{10}-120 a_{1}^{10} a_{3}^{2} a_{10}$

$\left.-240 a_{1}^{10} a_{2} a_{4} a_{10}+15 a_{1}^{11} a_{5} a_{10}+680 a_{1}^{9} a_{2}^{3} a_{11}-240 a_{1}^{10} a_{2} a_{3} a_{11}+15 a_{1}^{11} a_{4} a_{11}-120 a_{1}^{10} a_{2}^{2} a_{12}+15 a_{1}^{11} a_{3} a_{12}+15 a_{1}^{11} a_{2} a_{13}-a_{1}^{12} a_{14}\right)$

The resulting coefficients $B_{k}$ for $k=1,2,3$, and 4 are given by

$$
\begin{aligned}
& -B_{1}=A_{1}, \\
& -B_{3}=A_{3}+5 A_{1} A_{2}+5 A_{1}^{3}, \\
& -B_{2}=A_{2}+2 A_{1}^{2}, \\
& -B_{4}=A_{4}+6 A_{1} A_{3}+3 A_{2}^{2}+21 A_{1}^{2} A_{2}+14 A_{1}^{4} .
\end{aligned}
$$

These can be shown to be equivalent to (5) by setting $-B_{k}=$ $b_{k+1} a_{1}^{k+1}$ and $A_{k}=-a_{k+1} / a_{1}$. The coefficients $B_{k}$ for $k=$ $1,2, \ldots, 7$ can be found tabulated in [20], for $k=1,2, \ldots, 9$ in [21] and for $k=1,2, \ldots, 12$ they are tabulated in [22] with a different choice of the sign of $B_{k}$. Müller [23] has reported an alternative expression for $B_{k}$ and some symmetry relations for the coefficients. 


\section{Application to Mittag-Leffler Functions}

Many Mittag-Leffler functions can be represented in terms of elementary functions. For example,

$$
\begin{aligned}
E_{1,1}(-z)=\sum_{k=0}^{\infty} \frac{(-z)^{k}}{\Gamma(k+1)}=\operatorname{Exp}(-z) \\
E_{1 / 2,3}(-z)=\sum_{k=0}^{\infty} \frac{(-z)^{k}}{\Gamma(k / 2+3)} \\
=\frac{\operatorname{Exp}\left(z^{2}\right) \operatorname{erfc}(z)-1}{z^{4}}-\frac{1}{z^{2}}+\frac{4}{3 z \sqrt{\pi}}+\frac{2}{z^{3} \sqrt{\pi}} .
\end{aligned}
$$

Applying (10) from the above theory to these functions whose values can be determined as accurately as possible using their alternative representations yields

$$
\begin{gathered}
-z=E_{1,1}^{-1}(w)=(w-1)-\frac{1}{2}(w-1)^{2}+\frac{1}{3}(w-1)^{3} \\
-\frac{1}{4}(w-1)^{4}+\cdots=\sum_{k=1}^{\infty} \frac{(-1)^{k+1}(w-1)^{k}}{k} \\
-z=E_{1 / 2,3}^{-1}(w)= \\
\quad \frac{15 \sqrt{\pi}}{8}\left(w-\frac{1}{2}\right)-\frac{1125 \pi^{3 / 2}}{1024}\left(w-\frac{1}{2}\right)^{2} \\
+\frac{3375 \pi^{3 / 2}(175 \pi-256)}{458752}\left(w-\frac{1}{2}\right)^{3}+\cdots
\end{gathered}
$$

A few observations are in order. Equations (16) and (17) are typical of the inverse of most infinite series; that is, they are also infinite series and do not converge rapidly. This can be easily illustrated by the following examples. For $w=\operatorname{Exp}(-1)$, (16) should yield $-z=-1$ (equivalently $z=1$ ). However, (16) requires 20 terms before the value of $z$ is as large as 0.99999 (5 nines), 44 terms for 10 nines, 68 terms for 15 nines, and 92 terms for 20 nines. Whereas for $w=\operatorname{Exp}(-10)$, where (16) should yield $z=10,156995$ terms are required before the value of $z$ is as large as 9.9999 (5 nines), 391895 terms for 10 nines, 635259 for 15 nines, and 881815 terms for 20 nines. Similarly, for $w=\operatorname{Exp}(-15)$ where (16) should yield $z=15,16730862$ terms are required before the value of $z$ is as large as 14.999 (3 nines), 51041531 terms for 8 nines, 87009540 terms for 13 nines, and 123532970 terms for 18 nines. For $w=$ $\operatorname{Exp}(-z)$, as $z$ becomes large (or equivalently $w \rightarrow 0$ ), the number of terms in (16) required to yield a value accurate to a given number of significant digits becomes astronomically large.

A similar behavior is exhibited in (17). For $w=$

\begin{tabular}{|c|c|}
\hline$k$ & $b_{k}$ \\
\hline 1 & +0.93543756289254634824 \\
\hline 2 & +0.90975389394768139194 \\
\hline 3 & +0.90540301580659885103 \\
\hline 4 & +0.90454074680764978103 \\
\hline 5 & +0.90437439055401830557 \\
\hline 6 & +0.90434827833630659461 \\
\hline 7 & +0.90434699795298307168 \\
\hline 8 & +0.90434836056866111562 \\
\hline 9 & +0.90434917779666970118 \\
\hline 10 & +0.90434948952806441367 \\
\hline 11 & +0.90434957941529405394 \\
\hline 12 & +0.90434959664285150118 \\
\hline 13 & +0.90434959619870743701 \\
\hline 14 & +0.90434959373645610938 \\
\hline 15 & +0.90434959223258048967 \\
\hline 16 & +0.90434959159682667227 \\
\hline 17 & +0.90434959139076386922 \\
\hline 18 & +0.90434959134523887081 \\
\hline 19 & +0.90434959134495719874 \\
\hline 20 & +0.90434959135163221718 \\
\hline 21 & +0.90434959135628153678 \\
\hline 22 & +0.90434959135847703425 \\
\hline 23 & +0.90434959135928171696 \\
\hline 24 & +0.90434959135949807367 \\
\hline 25 & +0.90434959135952104631 \\
\hline 26 & +0.90434959135950171101 \\
\hline 27 & +0.90434959135948382131 \\
\hline 28 & +0.90434959135947403392 \\
\hline 29 & +0.90434959135946990785 \\
\hline 30 & +0.90434959135946855244 \\
\hline 31 & +0.90434959135946826616 \\
\hline 32 & +0.90434959135946829135 \\
\hline 33 & +0.90434959135946836127 \\
\hline 34 & +0.90434959135946840959 \\
\hline 35 & +0.90434959135946843362 \\
\hline 36 & +0.90434959135946844315 \\
\hline 37 & +0.90434959135946844604 \\
\hline 38 & +0.90434959135946844650 \\
\hline 39 & +0.90434959135946844632 \\
\hline 40 & +0.90434959135946844609 \\
\hline 41 & +0.90434959135946844595 \\
\hline 42 & +0.90434959135946844588 \\
\hline 43 & +0.90434959135946844585 \\
\hline 44 & +0.90434959135946844585 \\
\hline 45 & +0.90434959135946844584 \\
\hline 46 & +0.90434959135946844585 \\
\hline
\end{tabular}
$0.30821552131 \ldots$, (17) should yield $z=1$. To obtain a value of $z$ as large as 0.99999 ( 5 nines), 12 terms are required, 24 terms for 10 nines, 36 terms for 15 nines, and 48 terms for 20 nines. For $w=0.0662592710 \ldots$, (17) should yield $z=10$, but
TABLE 2: Coefficients $b_{k}$ for the inverse Mittag-Leffler function $-z=$ $E_{1 / 7,1}^{-1}(w)$.

81 terms are required to obtain a value of $z$ as large as 9.9999 (5 nines), 162 for 10 nines, 243 terms for 15 nines, and 324 terms for 20 nines. For $w=0.007423646216 \ldots$, (17) should 
TABLE 3: Number of terms required in the finite representation of $E_{\alpha, \beta}^{-1}(z)$ for 20-significant-digit accuracy.

\begin{tabular}{lcc}
\hline$\alpha$ & $\beta=1$ & $\beta=2$ \\
\hline $1 / 100$ & 11 & 9 \\
$1 / 10$ & 32 & 25 \\
$1 / 9$ & 35 & 27 \\
$1 / 8$ & 40 & 28 \\
$1 / 7$ & 46 & 33 \\
$1 / 6$ & 52 & 38 \\
$1 / 5$ & 64 & 46 \\
$1 / 4$ & 92 & 63 \\
$1 / 3$ & 156 & 91 \\
$1 / 2$ & 562 & 262 \\
$4 / 7$ & 1051 & 429 \\
$3 / 5$ & 1469 & 548 \\
\hline
\end{tabular}

TABLE 4: Index of Mittag-Leffler inverse examples.

\begin{tabular}{lcl}
\hline$\alpha$ & $\beta=1$ & $\beta=2$ \\
\hline $1 / 3$ & Equation (22) and Table 5 & Equation (29) and Table 12 \\
$1 / 4$ & Equation (23) and Table 6 & Equation (30) and Table 13 \\
$1 / 5$ & Equation (24) and Table 7 & Equation (31) and Table 14 \\
$1 / 6$ & Equation (25) and Table 8 & Equation (32) and Table 15 \\
$1 / 7$ & Equation (19) and Table 2 & Equation (33) and Table 16 \\
$1 / 8$ & Equation (26) and Table 9 & Equation (34) and Table 17 \\
$1 / 9$ & Equation (27) and Table 10 & Equation (35) and Table 18 \\
$1 / 10$ & Equation (28) and Table 11 & Equation (36) and Table 19 \\
\hline
\end{tabular}

yield $z=100$, but 770 terms are required to obtain a value of $z$ as large as 99.999 ( 5 nines) and 1540 terms for 10 nines. There is, however, one big difference between (16) and (17). Equation (16) is one of the few inverses of a Mittag-Leffler function, where the coefficients $b_{k}$ in the inverse given in (10) and itemized in Table 1 for $b_{1}-b_{14}$ simplify to a tractable expression; in this case $b_{k}=(-1)^{k+1} / k$. The mathematical manipulations required to obtain the coefficients $b_{k}$ in (17) using (10) become algebraically intensive as $k$ becomes large. Whereas $b_{14}$ given in Table 1 contains 101 terms, $b_{1000}$ contains more than $2.4 \times 10^{31}$ terms. Consequently, although the infinite series given in (17) correctly represents the inverse Mittag-Leffler function, it is impractical to use for anything other than small $z$ where only a reasonable number of terms are needed for the required accuracy. This is the case for most of the inverse Mittag-Leffler functions.

Consider the inverse of the Mittag-Leffler function $E_{1 / 7,1}(-z)$. The coefficients $b_{k}$ calculated from (10) are given in Table 2 (truncated to 20 significant digits).

It is obvious in looking at the coefficients $b_{k}$ in Table 2 that they are approaching a constant as $k$ becomes large. In this case, the constant is $1 / \Gamma(6 / 7)$. Subsequently, the first 20 significant digits for all coefficients after $b_{46}$ are identical
TABLE 5: Coefficients $b_{k}$ for the inverse Mittag-Leffler function $-z=$ $E_{1 / 3,1}^{-1}(w)$.

\begin{tabular}{|c|c|}
\hline$k$ & $b_{k}$ \\
\hline 1 & +0.89297951156924921122 \\
\hline 2 & +0.78878610417460496420 \\
\hline 3 & +0.75763354875769329328 \\
\hline 4 & +0.74579778773344787841 \\
\hline 5 & +0.74098130749558031810 \\
\hline 6 & +0.73904720310555344414 \\
\hline 7 & +0.73834522959265981505 \\
\hline 8 & +0.73816142472165138055 \\
\hline 9 & +0.73817790739448622896 \\
\hline 10 & +0.73825511074871374070 \\
\hline 11 & +0.73833571434942144250 \\
\hline 12 & +0.73839997927465491398 \\
\hline 13 & +0.73844449496113714586 \\
\hline 14 & +0.73847210628873099793 \\
\hline 15 & +0.73848730878287129409 \\
\hline 16 & +0.73849432235279002530 \\
\hline 17 & +0.73849644605470171587 \\
\hline 18 & +0.73849598893480155549 \\
\hline 19 & +0.73849442620461442205 \\
\hline 20 & +0.73849261331276901456 \\
\hline 21 & +0.73849098486630158241 \\
\hline 22 & +0.73848971269933349619 \\
\hline 23 & +0.73848881978568953731 \\
\hline 24 & +0.73848825605202219473 \\
\hline 25 & +0.73848794494184281702 \\
\hline 26 & +0.73848780930951379358 \\
\hline 27 & +0.73848778373908570968 \\
\hline 28 & +0.73848781862086802257 \\
\hline 29 & +0.73848787970874775507 \\
\hline 30 & +0.73848794558318757255 \\
\hline 31 & +0.73848800448141685314 \\
\hline 32 & +0.73848805128840606965 \\
\hline 33 & +0.73848808504905157547 \\
\hline 34 & +0.73848810710188503719 \\
\hline 35 & +0.73848811979412736597 \\
\hline 36 & +0.73848812567490815232 \\
\hline 37 & +0.73848812704699497915 \\
\hline 38 & +0.73848812576600764535 \\
\hline 39 & +0.73848812319593135382 \\
\hline 40 & +0.73848812025242302623 \\
\hline 41 & +0.73848811748639447885 \\
\hline 42 & +0.73848811517762375779 \\
\hline 43 & +0.73848811342117360995 \\
\hline 44 & +0.73848811219849196547 \\
\hline 45 & +0.73848811143091641775 \\
\hline 46 & +0.73848811101668555873 \\
\hline 47 & +0.73848811085420482928 \\
\hline 48 & +0.73848811085483076012 \\
\hline
\end{tabular}


TABLE 5: Continued.

\begin{tabular}{|c|c|}
\hline$k$ & $b_{k}$ \\
\hline 49 & +0.73848811094828991640 \\
\hline 50 & +0.73848811108336786221 \\
\hline 51 & +0.73848811122590646140 \\
\hline 52 & +0.73848811135556505150 \\
\hline 53 & +0.73848811146230003348 \\
\hline 54 & +0.73848811154312398420 \\
\hline 55 & +0.73848811159941978280 \\
\hline 56 & +0.73848811163489404655 \\
\hline 57 & +0.73848811165413834046 \\
\hline 58 & +0.73848811166170643685 \\
\hline 59 & +0.73848811166159371995 \\
\hline 60 & +0.73848811165700633342 \\
\hline 61 & +0.73848811165032209352 \\
\hline 62 & +0.73848811164316504107 \\
\hline 63 & +0.73848811163653598310 \\
\hline 64 & +0.73848811163095974872 \\
\hline 65 & +0.73848811162662488952 \\
\hline 66 & +0.73848811162350289072 \\
\hline 67 & +0.73848811162144189450 \\
\hline 68 & +0.73848811162023502242 \\
\hline 69 & +0.73848811161966626697 \\
\hline 70 & +0.73848811161953822181 \\
\hline 71 & +0.73848811161968617262 \\
\hline 72 & +0.73848811161998269778 \\
\hline 73 & +0.73848811162033624249 \\
\hline 74 & +0.73848811162068634439 \\
\hline 75 & +0.73848811162099743490 \\
\hline 76 & +0.73848811162125249050 \\
\hline 77 & +0.73848811162144729066 \\
\hline 78 & +0.73848811162158565606 \\
\hline 79 & +0.73848811162167577734 \\
\hline 80 & +0.73848811162172758004 \\
\hline 81 & +0.73848811162175098150 \\
\hline 82 & +0.73848811162175485904 \\
\hline 83 & +0.73848811162174654725 \\
\hline 84 & +0.73848811162173170072 \\
\hline 85 & +0.73848811162171438724 \\
\hline 86 & +0.73848811162169730770 \\
\hline 87 & +0.73848811162168206810 \\
\hline 88 & +0.73848811162166945421 \\
\hline 89 & +0.73848811162165967945 \\
\hline 90 & +0.73848811162165259141 \\
\hline 91 & +0.73848811162164783248 \\
\hline 92 & +0.73848811162164495684 \\
\hline 93 & +0.73848811162164350958 \\
\hline 94 & +0.73848811162164307502 \\
\hline 95 & +0.73848811162164330226 \\
\hline 96 & +0.73848811162164391339 \\
\hline 97 & +0.73848811162164470224 \\
\hline
\end{tabular}

TABle 5: Continued.

\begin{tabular}{|c|c|}
\hline$k$ & $b_{k}$ \\
\hline 98 & +0.73848811162164552587 \\
\hline 99 & +0.73848811162164629360 \\
\hline 100 & +0.73848811162164695521 \\
\hline 101 & +0.73848811162164748994 \\
\hline 102 & +0.73848811162164789692 \\
\hline 103 & +0.73848811162164818753 \\
\hline 104 & +0.73848811162164837957 \\
\hline 105 & +0.73848811162164849309 \\
\hline 106 & +0.73848811162164854766 \\
\hline 107 & +0.73848811162164856073 \\
\hline 108 & +0.73848811162164854687 \\
\hline 109 & +0.73848811162164851751 \\
\hline 110 & +0.73848811162164848112 \\
\hline 111 & +0.73848811162164844359 \\
\hline 112 & +0.73848811162164840865 \\
\hline 113 & +0.73848811162164837840 \\
\hline 114 & +0.73848811162164835374 \\
\hline 115 & +0.73848811162164833471 \\
\hline 116 & +0.73848811162164832086 \\
\hline 117 & +0.73848811162164831146 \\
\hline 118 & +0.73848811162164830565 \\
\hline 119 & +0.73848811162164830260 \\
\hline 120 & +0.73848811162164830154 \\
\hline 121 & +0.73848811162164830182 \\
\hline 122 & +0.73848811162164830293 \\
\hline 123 & +0.73848811162164830447 \\
\hline 124 & +0.73848811162164830614 \\
\hline 125 & +0.73848811162164830777 \\
\hline 126 & +0.73848811162164830923 \\
\hline 127 & +0.73848811162164831046 \\
\hline 128 & +0.73848811162164831145 \\
\hline 129 & +0.73848811162164831220 \\
\hline 130 & +0.73848811162164831273 \\
\hline 131 & +0.73848811162164831309 \\
\hline 132 & +0.73848811162164831331 \\
\hline 133 & +0.73848811162164831342 \\
\hline 134 & +0.73848811162164831345 \\
\hline 135 & +0.73848811162164831343 \\
\hline 136 & +0.73848811162164831338 \\
\hline 137 & +0.73848811162164831331 \\
\hline 138 & +0.73848811162164831323 \\
\hline 139 & +0.73848811162164831316 \\
\hline 140 & +0.73848811162164831310 \\
\hline 141 & +0.73848811162164831304 \\
\hline 142 & +0.73848811162164831300 \\
\hline 143 & +0.73848811162164831297 \\
\hline 144 & +0.73848811162164831294 \\
\hline 145 & +0.73848811162164831292 \\
\hline 146 & +0.73848811162164831291 \\
\hline
\end{tabular}


TABle 5: Continued.

\begin{tabular}{lc}
\hline$k$ & $b_{k}$ \\
\hline 147 & +0.73848811162164831291 \\
148 & +0.73848811162164831291 \\
149 & +0.73848811162164831291 \\
150 & +0.73848811162164831291 \\
151 & +0.73848811162164831291 \\
152 & +0.73848811162164831292 \\
153 & +0.73848811162164831292 \\
154 & +0.73848811162164831292 \\
155 & +0.73848811162164831292 \\
156 & +0.73848811162164831293 \\
\hline
\end{tabular}

differing only after the first 20 digits. Thus, applying (4) with $z_{o}=0, a_{o}=1$, the inverse for $E_{1 / 7,1}(-z)$ can be written as

$$
-z=E_{1 / 7,1}^{-1}(w)=-\sum_{k=1}^{46} b_{k}(1-w)^{k}-\sum_{k=47}^{\infty} \frac{(1-w)^{k}}{\Gamma(6 / 7)} .
$$

Equation (18) assumes that all coefficients $b_{k}$ for $k>46$ can be approximated by $1 / \Gamma(6 / 7)$. The approximation is valid provided that an answer accurate to no more than 20 significant digits is sufficient. The last term in (18) is a geometric series which can be replaced by its corresponding sum yielding

$$
-z=E_{1 / 7,1}^{-1}(w)=-\sum_{k=1}^{46} b_{k}(1-w)^{k}-\frac{1}{\Gamma(6 / 7)} \frac{(1-w)^{47}}{w}
$$

Equation (19) represents a finite series for the inverse MittagLeffler function for $w \leq 1$ or equivalently $-z \leq 0$ accurate to 20 significant digits. The series has been tested numerically and in all cases tested gives the correct answer to at least 20 significant digits $0 \geq-z<-\infty$ or equivalently $0<$ $w \leq 1$. This finite series representation of the inverse MittagLeffler function has at least 3 advantages over the infinite series representation: (1) the finite series greatly expedites the evaluation of the inverse, (2) it is not limited to small $|-z|$, and (3) there is no ambiguity concerning the number of terms needed in the series to obtain a required accuracy in the final answer.

Note that if the required accuracy is only 10 significant digits, the first 10 digits of the coefficients $b_{k}$ after $b_{17}$ are identical differing only after the first 10 digits. In this case, the equation for the inverse can be written as

$$
-z=E_{1 / 7,1}^{-1}(w)=-\sum_{k=1}^{17} b_{k}(1-w)^{k}-\frac{1}{\Gamma(6 / 7)} \frac{(1-w)^{18}}{w}
$$

The fact that the coefficients $b_{k}$ approached a constant as $k$ becomes large allowed the infinite series to be written as a finite series. For what other Mittag-Leffler functions do the coefficients in the inverse approach a constant?

\begin{tabular}{|c|c|}
\hline$k$ & $b_{k}$ \\
\hline 1 & 0.90640247705547707798 \\
\hline 2 & 0.84026894007589891391 \\
\hline 3 & 0.82351018992990700207 \\
\hline 4 & 0.81828957550795105766 \\
\hline 5 & 0.81660707076917509278 \\
\hline 6 & 0.81610221036029616113 \\
\hline 7 & 0.81598545033147361884 \\
\hline 8 & 0.81598379842062516042 \\
\hline 9 & 0.81600622129819404882 \\
\hline 10 & 0.81602620538545447950 \\
\hline 11 & 0.81603889114688882765 \\
\hline 12 & 0.81604558885224673170 \\
\hline 13 & 0.81604856137343865455 \\
\hline 14 & 0.81604957197906280172 \\
\hline 15 & 0.81604970482268525625 \\
\hline 16 & 0.81604953186530296411 \\
\hline 17 & 0.81604931188945210690 \\
\hline 18 & 0.81604913762546883873 \\
\hline 19 & 0.81604902563306262589 \\
\hline 20 & 0.81604896432490207114 \\
\hline 21 & 0.81604893634591082941 \\
\hline 22 & 0.81604892711529213868 \\
\hline 23 & 0.81604892680747229621 \\
\hline 24 & 0.81604892974411138067 \\
\hline 25 & 0.81604893311589531898 \\
\hline 26 & 0.81604893581695267902 \\
\hline 27 & 0.81604893761527447364 \\
\hline 28 & 0.81604893864655309011 \\
\hline 29 & 0.81604893914547956993 \\
\hline 30 & 0.81604893932600511441 \\
\hline 31 & 0.81604893934331182207 \\
\hline 32 & 0.81604893929407644732 \\
\hline 33 & 0.81604893923044766688 \\
\hline 34 & 0.81604893917568606393 \\
\hline 35 & 0.81604893913673190214 \\
\hline 36 & 0.81604893911270302848 \\
\hline 37 & 0.81604893909990779088 \\
\hline 38 & 0.81604893909439699044 \\
\hline 39 & 0.81604893909300259361 \\
\hline 40 & 0.81604893909356459299 \\
\hline 41 & 0.81604893909479532456 \\
\hline 42 & 0.81604893909603395768 \\
\hline 43 & 0.81604893909701259830 \\
\hline 44 & 0.81604893909767829618 \\
\hline 45 & 0.81604893909807584283 \\
\hline 46 & 0.81604893909827997471 \\
\hline 47 & 0.81604893909836178143 \\
\hline 48 & 0.81604893909837594019 \\
\hline
\end{tabular}

TABLE 6: Coefficients $b_{k}$ for the inverse Mittag-Leffler function $-z=$ $E_{1 / 4,1}^{-1}(w)$. 
TABLE 6: Continued.

\begin{tabular}{|c|c|}
\hline$k$ & $b_{k}$ \\
\hline 49 & 0.81604893909835900689 \\
\hline 50 & 0.81604893909833251654 \\
\hline 51 & 0.81604893909830737526 \\
\hline 52 & 0.81604893909828785893 \\
\hline 53 & 0.81604893909827462118 \\
\hline 54 & 0.81604893909826667548 \\
\hline 55 & 0.81604893909826254932 \\
\hline 56 & 0.81604893909826086189 \\
\hline 57 & 0.81604893909826054632 \\
\hline 58 & 0.81604893909826087970 \\
\hline 59 & 0.81604893909826142714 \\
\hline 60 & 0.81604893909826196124 \\
\hline 61 & 0.81604893909826238717 \\
\hline 62 & 0.81604893909826268505 \\
\hline 63 & 0.81604893909826287085 \\
\hline 64 & 0.81604893909826297283 \\
\hline 65 & 0.81604893909826301912 \\
\hline 66 & 0.81604893909826303243 \\
\hline 67 & 0.81604893909826302882 \\
\hline 68 & 0.81604893909826301846 \\
\hline 69 & 0.81604893909826300704 \\
\hline 70 & 0.81604893909826299725 \\
\hline 71 & 0.81604893909826298997 \\
\hline 72 & 0.81604893909826298513 \\
\hline 73 & 0.81604893909826298224 \\
\hline 74 & 0.81604893909826298074 \\
\hline 75 & 0.81604893909826298012 \\
\hline 76 & 0.81604893909826298002 \\
\hline 77 & 0.81604893909826298015 \\
\hline 78 & 0.81604893909826298038 \\
\hline 79 & 0.81604893909826298060 \\
\hline 80 & 0.81604893909826298078 \\
\hline 81 & 0.81604893909826298092 \\
\hline 82 & 0.81604893909826298100 \\
\hline 83 & 0.81604893909826298105 \\
\hline 84 & 0.81604893909826298108 \\
\hline 85 & 0.81604893909826298109 \\
\hline 86 & 0.81604893909826298109 \\
\hline 87 & 0.81604893909826298109 \\
\hline 88 & 0.81604893909826298109 \\
\hline 89 & 0.81604893909826298108 \\
\hline 90 & 0.81604893909826298108 \\
\hline 91 & 0.81604893909826298108 \\
\hline 92 & 0.81604893909826298107 \\
\hline
\end{tabular}

\section{Inverse Mittag-Leffler Functions for Which $b_{k}$ Approach a Constant}

Evaluation of great many inverse Mittag-Leffler functions reveals several important points. (1) It has been shown that the Mittag-Leffer function with these $\alpha$ and $\beta$ parameters,
TABLE 7: Coefficients $b_{k}$ for the inverse Mittag-Leffler function $-z=$ $E_{1 / 5,1}^{-1}(w)$.

\begin{tabular}{|c|c|}
\hline$k$ & $b_{k}$ \\
\hline 1 & +0.91816874239976061064 \\
\hline 2 & +0.87239815820597071525 \\
\hline 3 & +0.86241404655813210186 \\
\hline 4 & +0.85979515758722241525 \\
\hline 5 & +0.85910249498870304439 \\
\hline 6 & +0.85894043515591457893 \\
\hline 7 & +0.85891680448152655590 \\
\hline 8 & +0.85892230156211349359 \\
\hline 9 & +0.85892962600662844119 \\
\hline 10 & +0.85893406838573395241 \\
\hline 11 & +0.85893613453863527233 \\
\hline 12 & +0.85893690887578873062 \\
\hline 13 & +0.85893711768226131089 \\
\hline 14 & +0.85893712714123260150 \\
\hline 15 & +0.85893708951453251359 \\
\hline 16 & +0.85893705517782142799 \\
\hline 17 & +0.85893703407253609369 \\
\hline 18 & +0.85893702365426492211 \\
\hline 19 & +0.85893701950167094637 \\
\hline 20 & +0.85893701834496455469 \\
\hline 21 & +0.85893701834146563837 \\
\hline 22 & +0.85893701863535859269 \\
\hline 23 & +0.85893701890846377513 \\
\hline 24 & +0.85893701908567480745 \\
\hline 25 & +0.85893701917914447317 \\
\hline 26 & +0.85893701921959129502 \\
\hline 27 & +0.85893701923250482423 \\
\hline 28 & +0.85893701923367892385 \\
\hline 29 & +0.85893701923119867846 \\
\hline 30 & +0.85893701922846356042 \\
\hline 31 & +0.85893701922650554665 \\
\hline 32 & +0.85893701922537418952 \\
\hline 33 & +0.85893701922482812463 \\
\hline 34 & +0.85893701922461848499 \\
\hline 35 & +0.85893701922457079400 \\
\hline 36 & +0.85893701922458522240 \\
\hline 37 & +0.85893701922461381792 \\
\hline 38 & +0.85893701922463819190 \\
\hline 39 & +0.85893701922465413251 \\
\hline 40 & +0.85893701922466286636 \\
\hline 41 & +0.85893701922466687324 \\
\hline 42 & +0.85893701922466827905 \\
\hline 43 & +0.85893701922466848269 \\
\hline 44 & +0.85893701922466825732 \\
\hline 45 & +0.85893701922466796298 \\
\hline 46 & +0.85893701922466773028 \\
\hline 47 & +0.85893701922466758222 \\
\hline 48 & +0.85893701922466750209 \\
\hline
\end{tabular}


TABLE 7: Continued.

\begin{tabular}{lc}
\hline$k$ & $b_{k}$ \\
\hline 49 & +0.85893701922466746565 \\
50 & +0.85893701922466745311 \\
51 & +0.85893701922466745162 \\
52 & +0.85893701922466745405 \\
53 & +0.85893701922466745708 \\
54 & +0.85893701922466745948 \\
55 & +0.85893701922466746103 \\
56 & +0.85893701922466746188 \\
57 & +0.85893701922466746229 \\
58 & +0.85893701922466746243 \\
59 & +0.85893701922466746246 \\
60 & +0.85893701922466746244 \\
61 & +0.85893701922466746241 \\
62 & +0.85893701922466746238 \\
63 & +0.85893701922466746236 \\
64 & +0.85893701922466746235 \\
\hline
\end{tabular}

namely, $0<\alpha<1$ and $\beta>\alpha$, is a completely monotonic decreasing function $[24,25]$, and thus the inverse is guaranteed to be single valued. (2) The coefficients $b_{k}$ in the inverse approach a constant only when the parameter $\beta$ is either 1 or 2 . (3) The coefficients $b_{k}$ approach a constant only when the parameter $\alpha<1$. (4) The coefficients $b_{k}$ approach a constant given by

$$
\lim _{k \rightarrow \infty} b_{k}=\frac{1}{\Gamma(\beta-\alpha)} .
$$

Consequently, as $\alpha \rightarrow 0$, the coefficient $b_{k} \rightarrow 1$ for both $\beta=1$ and 2. However, for $\beta=1$ the coefficient $b_{k}$ is always less than 1 while for $\beta=2, b_{k}$ is always greater than 1 as $\alpha \rightarrow 0$. (5) The smaller the value of $\alpha$, the fewer the numerical terms required in the inverse series to obtain a given significant digit accuracy. This is illustrated in Table 3 which gives the number of terms required in the finite representation of the inverse Mittag-Leffler function for 20-significant-digit accuracy for various values of $\alpha$ with $\beta=1$ and $\beta=2$.

Extending this logic to its natural conclusion implies that at $\alpha=0$ no terms will be required in the series. To see that this is correct, note that using (1) both $w=E_{0,1}(-z)$ and $w=$ $E_{0,2}(-z)$ reduce to $w=1 /(1+z)$ when $\alpha=0$. Inverting and solving for $-z$ yield $-z=-(1-w) / w$. This is consistent with (19) which reduces to this same result when the upper limit on the summation is $k=0$ (no terms in the summation) and the factor $1 / \Gamma(6 / 7)$ is replaced by the more general equation (21) which gives unity for $\alpha=0$ and $\beta=1$ or $\beta=2$.

Conversely, as $\alpha$ approaches 1 , an increasingly larger number of numerical terms are required in the inverse series to obtain a given significant digit accuracy as Table 3 illustrates. (6) Consequently, as $\alpha$ increases above $1 / 2$, the inverse Mittag-Leffler function described by a finite series requires more and more terms becoming less practical. For example, for $\alpha=0.74$ and $\beta=1$, for $b_{k}$ to converge to just 5 significant digits requires 2215 terms while, for $\alpha=0.825$ and $\beta=2$, requiring 1828 terms for the same convergence.
TABLE 8: Coefficients $b_{k}$ for the inverse Mittag-Leffler function $-z=$ $E_{1 / 6,1}^{-1}(w)$.

\begin{tabular}{|c|c|}
\hline$k$ & $b_{k}$ \\
\hline 1 & +0.92771933363003920070 \\
\hline 2 & +0.89414577241424278746 \\
\hline 3 & +0.88773763213642664587 \\
\hline 4 & +0.88628977973846244289 \\
\hline 5 & +0.88596635420228565868 \\
\hline 6 & +0.88590523526021224793 \\
\hline 7 & +0.88589982030554044972 \\
\hline 8 & +0.88590275825406298752 \\
\hline 9 & +0.88590511036557706322 \\
\hline 10 & +0.88590621213903655148 \\
\hline 11 & +0.88590660928393383455 \\
\hline 12 & +0.88590671636180511607 \\
\hline 13 & +0.88590672946995710075 \\
\hline 14 & +0.88590672153714995480 \\
\hline 15 & +0.88590671360108075777 \\
\hline 16 & +0.88590670912379822800 \\
\hline 17 & +0.88590670718459732010 \\
\hline 18 & +0.88590670653013358196 \\
\hline 19 & +0.88590670638937664049 \\
\hline 20 & +0.88590670640476657586 \\
\hline 21 & +0.88590670644420859302 \\
\hline 22 & +0.88590670647244801433 \\
\hline 23 & +0.88590670648712373933 \\
\hline 24 & +0.88590670649321013736 \\
\hline 25 & +0.88590670649512780068 \\
\hline 26 & +0.88590670649542543061 \\
\hline 27 & +0.88590670649526508181 \\
\hline 28 & +0.88590670649506835081 \\
\hline 29 & +0.88590670649493875951 \\
\hline 30 & +0.88590670649487287445 \\
\hline 31 & +0.88590670649484578753 \\
\hline 32 & +0.88590670649483743413 \\
\hline 33 & +0.88590670649483637544 \\
\hline 34 & +0.88590670649483735333 \\
\hline 35 & +0.88590670649483843570 \\
\hline 36 & +0.88590670649483914752 \\
\hline 37 & +0.88590670649483951597 \\
\hline 38 & +0.88590670649483967198 \\
\hline 39 & +0.88590670649483972248 \\
\hline 40 & +0.88590670649483973021 \\
\hline 41 & +0.88590670649483972509 \\
\hline 42 & +0.88590670649483971872 \\
\hline 43 & +0.88590670649483971430 \\
\hline 44 & +0.88590670649483971188 \\
\hline 45 & +0.88590670649483971080 \\
\hline 46 & +0.88590670649483971040 \\
\hline 47 & +0.88590670649483971032 \\
\hline 48 & +0.88590670649483971034 \\
\hline
\end{tabular}


TABLE 8: Continued.

\begin{tabular}{lc}
\hline$k$ & $b_{k}$ \\
\hline 49 & +0.88590670649483971037 \\
50 & +0.88590670649483971040 \\
51 & +0.88590670649483971042 \\
52 & +0.88590670649483971043 \\
\hline
\end{tabular}

TABLE 9: Coefficients $b_{k}$ for the inverse Mittag-Leffler function $-z=$ $E_{1 / 8,1}^{-1}(w)$.

\begin{tabular}{|c|c|}
\hline$k$ & $b_{k}$ \\
\hline 1 & +0.94174269984970148808 \\
\hline 2 & +0.92145833616345434435 \\
\hline 3 & +0.91837205036733785079 \\
\hline 4 & +0.91782768940815950206 \\
\hline 5 & +0.91773538138444524775 \\
\hline 6 & +0.91772307499596115692 \\
\hline 7 & +0.91772281742094063440 \\
\hline 8 & +0.91772345536444575339 \\
\hline 9 & +0.91772376557618704885 \\
\hline 10 & +0.91772386504763452141 \\
\hline 11 & +0.91772388849585357984 \\
\hline 12 & +0.91772389156330350231 \\
\hline 13 & +0.91772389092194344445 \\
\hline 14 & +0.91772389025312412600 \\
\hline 15 & +0.91772388994408363001 \\
\hline 16 & +0.91772388984022900150 \\
\hline 17 & +0.91772388981468002747 \\
\hline 18 & +0.91772388981165650624 \\
\hline 19 & +0.91772388981289452457 \\
\hline 20 & +0.91772388981401655984 \\
\hline 21 & +0.91772388981455209678 \\
\hline 22 & +0.91772388981474237399 \\
\hline 23 & +0.91772388981479260777 \\
\hline 24 & +0.91772388981479946414 \\
\hline 25 & +0.91772388981479716385 \\
\hline 26 & +0.91772388981479476203 \\
\hline 27 & +0.91772388981479352017 \\
\hline 28 & +0.91772388981479304158 \\
\hline 29 & +0.91772388981479290058 \\
\hline 30 & +0.91772388981479287486 \\
\hline 31 & +0.91772388981479287784 \\
\hline 32 & +0.91772388981479288351 \\
\hline 33 & +0.91772388981479288690 \\
\hline 34 & +0.91772388981479288835 \\
\hline 35 & +0.91772388981479288884 \\
\hline 36 & +0.91772388981479288896 \\
\hline 37 & +0.91772388981479288897 \\
\hline 38 & +0.91772388981479288896 \\
\hline 39 & +0.91772388981479288895 \\
\hline 40 & +0.91772388981479288894 \\
\hline
\end{tabular}

TABLE 10: Coefficients $b_{k}$ for the inverse Mittag-Leffler function $-z=$ $E_{1 / 9,1}^{-1}(w)$.

\begin{tabular}{|c|c|}
\hline$k$ & $b_{k}$ \\
\hline 1 & 0.94696534880216399450 \\
\hline 2 & 0.93053890407728875727 \\
\hline 3 & 0.92827170167793346183 \\
\hline 4 & 0.92791158668373485876 \\
\hline 5 & 0.92785715845937955393 \\
\hline 6 & 0.92785088338236675169 \\
\hline 7 & 0.92785088003507065240 \\
\hline 8 & 0.92785119095520099570 \\
\hline 9 & 0.92785131874466606751 \\
\hline 10 & 0.92785135396452045173 \\
\hline 11 & 0.92785136086263488623 \\
\hline 12 & 0.92785136142419633245 \\
\hline 13 & 0.92785136114025016525 \\
\hline 14 & 0.92785136095166820529 \\
\hline 15 & 0.92785136088086115418 \\
\hline 16 & 0.92785136086138201458 \\
\hline 17 & 0.92785136085777479380 \\
\hline 18 & 0.92785136085772523465 \\
\hline 19 & 0.92785136085805437723 \\
\hline 20 & 0.92785136085823967523 \\
\hline 21 & 0.92785136085830846162 \\
\hline 22 & 0.92785136085832754930 \\
\hline 23 & 0.92785136085833100710 \\
\hline 24 & 0.92785136085833091281 \\
\hline 25 & 0.92785136085833047865 \\
\hline 26 & 0.92785136085833023736 \\
\hline 27 & 0.92785136085833014532 \\
\hline 28 & 0.92785136085833011870 \\
\hline 29 & 0.92785136085833011354 \\
\hline 30 & 0.92785136085833011358 \\
\hline 31 & 0.92785136085833011420 \\
\hline 32 & 0.92785136085833011458 \\
\hline 33 & 0.92785136085833011473 \\
\hline 34 & 0.92785136085833011478 \\
\hline 35 & 0.92785136085833011479 \\
\hline
\end{tabular}

(7) For the same $\alpha$, the number of terms in the inverse for a desired accuracy is less for $\beta=2$ than for $\beta=1$. (8) According to $(21)$, when $\alpha=1$ and $\beta=1$, the coefficients $b_{k}$ in the inverse for the Mittag-Leffler function $E_{1,1}(-z)$ approach the constant zero as $k \rightarrow \infty$ as seen in (16) while for $\alpha=1$ and $\beta=2$ the coefficients $b_{k}$ in the inverse for the Mittag-Leffler function $E_{1,2}(-z)$ approach 1 as $k \rightarrow \infty$. (9) As noted above, according to (21), for $\beta=2$ the coefficients $b_{k}$ as $k \rightarrow \infty$ approach 1 as $\alpha \rightarrow 0$ and as $\alpha \rightarrow 1$ and $b_{k}$ is greater than 1 for $0<\alpha<1$. This implies that there exists a relative maximum value of $b_{k}$ as $k \rightarrow \infty$ in the range $0<\alpha<1$. This maximum occurs at $\alpha=0.5383678550 \ldots$ and corresponds to $b_{k}=1.129173885 \ldots$ as $k \rightarrow \infty$. Illustrating the above observations are numerous examples in the next section. 
TABLE 11: Coefficients $b_{k}$ for the inverse Mittag-Leffler function $-z=$ $E_{1 / 10,1}^{-1}(w)$.

\begin{tabular}{|c|c|}
\hline$k$ & $b_{k}$ \\
\hline 1 & +0.95135076986687318362 \\
\hline 2 & +0.93777687277778653379 \\
\hline 3 & +0.93606310059788083658 \\
\hline 4 & +0.93581557876046714164 \\
\hline 5 & +0.93578185282959558380 \\
\hline 6 & +0.93577844037062189666 \\
\hline 7 & +0.93577848991569929215 \\
\hline 8 & +0.93577864855859718780 \\
\hline 9 & +0.93577870513836571828 \\
\hline 10 & +0.93577871876872861069 \\
\hline 11 & +0.93577872101504047108 \\
\hline 12 & +0.93577872110471635717 \\
\hline 13 & +0.93577872099247192203 \\
\hline 14 & +0.93577872093543535964 \\
\hline 15 & +0.93577872091738487402 \\
\hline 16 & +0.93577872091324677459 \\
\hline 17 & +0.93577872091268732351 \\
\hline 18 & +0.93577872091275010310 \\
\hline 19 & +0.93577872091282731969 \\
\hline 20 & +0.93577872091286047983 \\
\hline 21 & +0.93577872091287052264 \\
\hline 22 & +0.93577872091287272009 \\
\hline 23 & +0.93577872091287295145 \\
\hline 24 & +0.93577872091287287167 \\
\hline 25 & +0.93577872091287280852 \\
\hline 26 & +0.93577872091287278263 \\
\hline 27 & +0.93577872091287277485 \\
\hline 28 & +0.93577872091287277316 \\
\hline 29 & +0.93577872091287277300 \\
\hline 30 & +0.93577872091287277308 \\
\hline 31 & +0.93577872091287277314 \\
\hline 32 & +0.93577872091287277317 \\
\hline
\end{tabular}

\section{Results for Specific $\alpha$ and $\beta$}

In this section, specific examples of various inverse MittagLeffler functions calculated using (10) will be given. Since the number of terms in the finite series for the inverse increases dramatically for $\alpha \geq 1 / 2$, then all examples will be for $\alpha<1 / 2$. All equations for the inverses are written assuming a desired 20-significant-digit accuracy. This is far greater accuracy than most requirements might call for; however, the equations can then be easily modified to any degree of accuracy less than 20 as outlined in the discussion of (20). Each Mittag-Leffler inverse $-z=E_{\alpha, \beta}^{-1}(w)$ example includes the equation of the form given in (19) valid for $0 \geq-z<-\infty$ (equivalently $0<w \leq 1$ ) representing the finite series representation of the inverse and a table with the corresponding coefficients $b_{k}$ truncated to 20 significant digits. The specific values of $\alpha$ and $\beta$ in each example are itemized in Table 4 which includes references to the
TABLE 12: Coefficients $b_{k}$ for the inverse Mittag-Leffler function $-z=$ $E_{1 / 3,2}^{-1}(w)$.

\begin{tabular}{|c|c|}
\hline$k$ & $b_{k}$ \\
\hline 1 & 1.1906393487589989482 \\
\hline 2 & 1.1218291259372159490 \\
\hline 3 & 1.1091651079345480360 \\
\hline 4 & 1.1070518842541741977 \\
\hline 5 & 1.1071094825114241570 \\
\hline 6 & 1.1074303857224016589 \\
\hline 7 & 1.1076404430424683811 \\
\hline 8 & 1.1077314700191564271 \\
\hline 9 & 1.1077553955944287187 \\
\hline 10 & 1.1077524578488089226 \\
\hline 11 & 1.1077434947715842855 \\
\hline 12 & 1.1077363499227504156 \\
\hline 13 & 1.1077324896384468086 \\
\hline 14 & 1.1077310965875528477 \\
\hline 15 & 1.1077310087501244588 \\
\hline 16 & 1.1077313864890338427 \\
\hline 17 & 1.1077317908641062832 \\
\hline 18 & 1.1077320654748424695 \\
\hline 19 & 1.1077322009818539445 \\
\hline 20 & 1.1077322408861236149 \\
\hline 21 & 1.1077322323951713722 \\
\hline 22 & 1.1077322084738571023 \\
\hline 23 & 1.1077321862732534401 \\
\hline 24 & 1.1077321716839450156 \\
\hline 25 & 1.1077321645662504099 \\
\hline 26 & 1.1077321625611127907 \\
\hline 27 & 1.1077321631931161511 \\
\hline 28 & 1.1077321646957392674 \\
\hline 29 & 1.1077321661093029166 \\
\hline 30 & 1.1077321670760136784 \\
\hline 31 & 1.1077321675814921360 \\
\hline 32 & 1.1077321677525601222 \\
\hline 33 & 1.1077321677362930981 \\
\hline 34 & 1.1077321676460497809 \\
\hline 35 & 1.1077321675494545998 \\
\hline 36 & 1.1077321674764692276 \\
\hline 37 & 1.1077321674332138537 \\
\hline 38 & 1.1077321674142290519 \\
\hline 39 & 1.1077321674107002345 \\
\hline 40 & 1.1077321674147729438 \\
\hline 41 & 1.1077321674210887571 \\
\hline 42 & 1.1077321674267605861 \\
\hline 43 & 1.1077321674307081625 \\
\hline 44 & 1.1077321674329036944 \\
\hline 45 & 1.1077321674337785530 \\
\hline 46 & 1.1077321674338524401 \\
\hline 47 & 1.1077321674335523501 \\
\hline 48 & 1.1077321674331583587 \\
\hline
\end{tabular}


TABle 12: Continued.

\begin{tabular}{|c|c|}
\hline$k$ & $b_{k}$ \\
\hline 49 & 1.1077321674328171346 \\
\hline 50 & 1.1077321674325809580 \\
\hline 51 & 1.1077321674324479205 \\
\hline 52 & 1.1077321674323926738 \\
\hline 53 & 1.1077321674323855472 \\
\hline 54 & 1.1077321674324019711 \\
\hline 55 & 1.1077321674324254123 \\
\hline 56 & 1.1077321674324467750 \\
\hline 57 & 1.1077321674324623978 \\
\hline 58 & 1.1077321674324719027 \\
\hline 59 & 1.1077321674324764817 \\
\hline 60 & 1.1077321674324777720 \\
\hline 61 & 1.1077321674324772490 \\
\hline 62 & 1.1077321674324759894 \\
\hline 63 & 1.1077321674324746516 \\
\hline 64 & 1.1077321674324735591 \\
\hline 65 & 1.1077321674324728113 \\
\hline 66 & 1.1077321674324723822 \\
\hline 67 & 1.1077321674324721933 \\
\hline 68 & 1.1077321674324721578 \\
\hline 69 & 1.1077321674324722031 \\
\hline 70 & 1.1077321674324722781 \\
\hline 71 & 1.1077321674324723525 \\
\hline 72 & 1.1077321674324724118 \\
\hline 73 & 1.1077321674324724522 \\
\hline 74 & 1.1077321674324724754 \\
\hline 75 & 1.1077321674324724857 \\
\hline 76 & 1.1077321674324724876 \\
\hline 77 & 1.1077321674324724851 \\
\hline 78 & 1.1077321674324724809 \\
\hline 79 & 1.1077321674324724766 \\
\hline 80 & 1.1077321674324724731 \\
\hline 81 & 1.1077321674324724707 \\
\hline 82 & 1.1077321674324724692 \\
\hline 83 & 1.1077321674324724685 \\
\hline 84 & 1.1077321674324724683 \\
\hline 85 & 1.1077321674324724684 \\
\hline 86 & 1.1077321674324724686 \\
\hline 87 & 1.1077321674324724688 \\
\hline 88 & 1.1077321674324724691 \\
\hline 89 & 1.1077321674324724692 \\
\hline 90 & 1.1077321674324724693 \\
\hline 91 & 1.1077321674324724694 \\
\hline
\end{tabular}

corresponding equations and table numbers for each example inverse.

For $\alpha=1 / 3$ and $\beta=1$, the equation for the inverse is given by

$$
-z=E_{1 / 3,1}^{-1}(w)=-\sum_{k=1}^{156} b_{k}(1-w)^{k}-\frac{1}{\Gamma(2 / 3)} \frac{(1-w)^{157}}{w}
$$

TABLE 13: Coefficients $b_{k}$ for the inverse Mittag-Leffler function $-z=$ $E_{1 / 4,2}^{-1}(w)$.

\begin{tabular}{|c|c|}
\hline$k$ & $b_{k}$ \\
\hline 1 & 1.1330030963193463474 \\
\hline 2 & 1.0941001823904933774 \\
\hline 3 & 1.0884969259036715641 \\
\hline 4 & 1.0878558386282093564 \\
\hline 5 & 1.0879299260072536857 \\
\hline 6 & 1.0880196901607404297 \\
\hline 7 & 1.0880580603477134188 \\
\hline 8 & 1.0880679256317365431 \\
\hline 9 & 1.0880681582727259222 \\
\hline 10 & 1.0880666922539625425 \\
\hline 11 & 1.0880656824079111948 \\
\hline 12 & 1.0880652661105853859 \\
\hline 13 & 1.0880651718209129462 \\
\hline 14 & 1.0880651893915501135 \\
\hline 15 & 1.0880652225919339689 \\
\hline 16 & 1.0880652438081294238 \\
\hline 17 & 1.0880652525894990987 \\
\hline 18 & 1.0880652545116835036 \\
\hline 19 & 1.0880652539426656281 \\
\hline 20 & 1.0880652530160160191 \\
\hline 21 & 1.0880652524013356965 \\
\hline 22 & 1.0880652521279001619 \\
\hline 23 & 1.0880652520573054501 \\
\hline 24 & 1.0880652520688854981 \\
\hline 25 & 1.0880652520973313877 \\
\hline 26 & 1.0880652521186658457 \\
\hline 27 & 1.0880652521294678814 \\
\hline 28 & 1.0880652521330822285 \\
\hline 29 & 1.0880652521332880539 \\
\hline 30 & 1.0880652521324663677 \\
\hline 31 & 1.0880652521316757550 \\
\hline 32 & 1.0880652521311980579 \\
\hline 33 & 1.0880652521309915951 \\
\hline 34 & 1.0880652521309409874 \\
\hline 35 & 1.0880652521309543925 \\
\hline 36 & 1.0880652521309814562 \\
\hline 37 & 1.0880652521310025596 \\
\hline 38 & 1.0880652521310142006 \\
\hline 39 & 1.0880652521310188181 \\
\hline 40 & 1.0880652521310196801 \\
\hline 41 & 1.0880652521310191068 \\
\hline 42 & 1.0880652521310183025 \\
\hline 43 & 1.0880652521310177100 \\
\hline 44 & 1.0880652521310173883 \\
\hline 45 & 1.0880652521310172608 \\
\hline 46 & 1.0880652521310172369 \\
\hline 47 & 1.0880652521310172534 \\
\hline 48 & 1.0880652521310172768 \\
\hline
\end{tabular}


TABLE 13: Continued.

\begin{tabular}{lc}
\hline$k$ & $b_{k}$ \\
\hline 49 & 1.0880652521310172946 \\
50 & 1.0880652521310173047 \\
51 & 1.0880652521310173090 \\
52 & 1.0880652521310173101 \\
53 & 1.0880652521310173098 \\
54 & 1.0880652521310173091 \\
55 & 1.0880652521310173086 \\
56 & 1.0880652521310173082 \\
57 & 1.0880652521310173081 \\
58 & 1.0880652521310173080 \\
59 & 1.0880652521310173080 \\
60 & 1.0880652521310173080 \\
61 & 1.0880652521310173080 \\
62 & 1.0880652521310173080 \\
63 & 1.0880652521310173081 \\
\hline
\end{tabular}

where $1 / \Gamma(2 / 3)=0.73848811162164831293 \ldots$ and the coefficients $b_{k}$ are given in Table 5 .

For $\alpha=1 / 4$ and $\beta=1$, the equation for the inverse is given by

$$
-z=E_{1 / 4,1}^{-1}(w)=-\sum_{k=1}^{92} b_{k}(1-w)^{k}-\frac{1}{\Gamma(3 / 4)} \frac{(1-w)^{93}}{w}
$$

where $1 / \Gamma(3 / 4)=0.81604893909826298107 \ldots$ and the coefficients $b_{k}$ are given in Table 6 .

For $\alpha=1 / 5$ and $\beta=1$, the equation for the inverse is given by

$$
-z=E_{1 / 5,1}^{-1}(w)=-\sum_{k=1}^{64} b_{k}(1-w)^{k}-\frac{1}{\Gamma(4 / 5)} \frac{(1-w)^{65}}{w}
$$

where $1 / \Gamma(4 / 5)=0.85893701922466746235 \ldots$ and the coefficients $b_{k}$ are given in Table 7 .

For $\alpha=1 / 6$ and $\beta=1$, the equation for the inverse is given by

$$
-z=E_{1 / 6,1}^{-1}(w)=-\sum_{k=1}^{52} b_{k}(1-w)^{k}-\frac{1}{\Gamma(5 / 6)} \frac{(1-w)^{53}}{w}
$$

where $1 / \Gamma(5 / 6)=0.88590670649483971043 \ldots$ and the coefficients $b_{k}$ are given in Table 8 .

For $\alpha=1 / 8$ and $\beta=1$, the equation for the inverse is given by

$$
-z=E_{1 / 8,1}^{-1}(w)=-\sum_{k=1}^{40} b_{k}(1-w)^{k}-\frac{1}{\Gamma(7 / 8)} \frac{(1-w)^{41}}{w}
$$

where $1 / \Gamma(7 / 8)=0.91772388981479288894 \ldots$ and the

\begin{tabular}{|c|c|}
\hline$k$ & $b_{k}$ \\
\hline 1 & 1.1018024908797127327 \\
\hline 2 & 1.0767885838427981399 \\
\hline 3 & 1.0738409976565079202 \\
\hline 4 & 1.0735918587371880661 \\
\hline 5 & 1.0736317220842957450 \\
\hline 6 & 1.0736613247947013790 \\
\hline 7 & 1.0736705276938335590 \\
\hline 8 & 1.0736719763838959650 \\
\hline 9 & 1.0736717194169915685 \\
\hline 10 & 1.0736714224350730386 \\
\hline 11 & 1.0736712961819915731 \\
\hline 12 & 1.0736712656573585536 \\
\hline 13 & 1.0736712658337183805 \\
\hline 14 & 1.0736712703837243345 \\
\hline 15 & 1.0736712730893571885 \\
\hline 16 & 1.0736712740367576320 \\
\hline 17 & 1.0736712741927198324 \\
\hline 18 & 1.0736712741349301121 \\
\hline 19 & 1.0736712740710214284 \\
\hline 20 & 1.0736712740390593289 \\
\hline 21 & 1.0736712740290815387 \\
\hline 22 & 1.0736712740281108537 \\
\hline 23 & 1.0736712740292769055 \\
\hline 24 & 1.0736712740302628755 \\
\hline 25 & 1.0736712740307308734 \\
\hline 26 & 1.0736712740308721433 \\
\hline 27 & 1.0736712740308820173 \\
\hline 28 & 1.0736712740308614201 \\
\hline 29 & 1.0736712740308445464 \\
\hline 30 & 1.0736712740308363495 \\
\hline 31 & 1.0736712740308337294 \\
\hline 32 & 1.0736712740308334598 \\
\hline 33 & 1.0736712740308337982 \\
\hline 34 & 1.0736712740308341087 \\
\hline 35 & 1.0736712740308342730 \\
\hline 36 & 1.0736712740308343326 \\
\hline 37 & 1.0736712740308343434 \\
\hline 38 & 1.0736712740308343390 \\
\hline 39 & 1.0736712740308343332 \\
\hline 40 & 1.0736712740308343296 \\
\hline 41 & 1.0736712740308343281 \\
\hline 42 & 1.0736712740308343277 \\
\hline 43 & 1.0736712740308343277 \\
\hline 44 & 1.0736712740308343278 \\
\hline 45 & 1.0736712740308343278 \\
\hline 46 & 1.0736712740308343279 \\
\hline
\end{tabular}
coefficients $b_{k}$ are given in Table 9.
TABLE 14: Coefficients $b_{k}$ for the inverse Mittag-Leffler function $-z=$ $E_{1 / 5,2}^{-1}(w)$. 
TABLE 15: Coefficients $b_{k}$ for the inverse Mittag-Leffler function $-z=$ $E_{1 / 6,2}^{-1}(w)$.

\begin{tabular}{|c|c|}
\hline$k$ & $b_{k}$ \\
\hline 1 & 1.0823392225683790674 \\
\hline 2 & 1.0649027775627960975 \\
\hline 3 & 1.0631672235921470234 \\
\hline 4 & 1.0630530019556810014 \\
\hline 5 & 1.0630738792005841325 \\
\hline 6 & 1.0630852477868224812 \\
\hline 7 & 1.0630879790229538911 \\
\hline 8 & 1.0630882395356188174 \\
\hline 9 & 1.0630881350924325915 \\
\hline 10 & 1.0630880687069684272 \\
\hline 11 & 1.0630880487821691319 \\
\hline 12 & 1.0630880460752193502 \\
\hline 13 & 1.0630880468406782122 \\
\hline 14 & 1.0630880475089981383 \\
\hline 15 & 1.0630880477592756779 \\
\hline 16 & 1.0630880478102509927 \\
\hline 17 & 1.0630880478069487489 \\
\hline 18 & 1.0630880477988781918 \\
\hline 19 & 1.0630880477949003960 \\
\hline 20 & 1.0630880477937454972 \\
\hline 21 & 1.0630880477936234680 \\
\hline 22 & 1.0630880477937097855 \\
\hline 23 & 1.0630880477937763019 \\
\hline 24 & 1.0630880477938030729 \\
\hline 25 & 1.0630880477938094515 \\
\hline 26 & 1.0630880477938094111 \\
\hline 27 & 1.0630880477938084584 \\
\hline 28 & 1.0630880477938078846 \\
\hline 29 & 1.0630880477938076743 \\
\hline 30 & 1.0630880477938076305 \\
\hline 31 & 1.0630880477938076352 \\
\hline 32 & 1.0630880477938076449 \\
\hline 33 & 1.0630880477938076504 \\
\hline 34 & 1.0630880477938076523 \\
\hline 35 & 1.0630880477938076527 \\
\hline 36 & 1.0630880477938076527 \\
\hline 37 & 1.0630880477938076526 \\
\hline 38 & 1.0630880477938076525 \\
\hline
\end{tabular}

For $\alpha=1 / 9$ and $\beta=1$, the equation for the inverse is given by

$$
-z=E_{1 / 9,1}^{-1}(w)=-\sum_{k=1}^{35} b_{k}(1-w)^{k}-\frac{1}{\Gamma(8 / 9)} \frac{(1-w)^{36}}{w}
$$

where $1 / \Gamma(8 / 9)=0.92785136085833011479 \ldots$ and the coefficients $b_{k}$ are given in Table 10 .
TABLE 16: Coefficients $b_{k}$ for the inverse Mittag-Leffler function $-z=$ $E_{1 / 7,2}^{-1}(w)$.

\begin{tabular}{lc}
\hline$k$ & $b_{k}$ \\
\hline 1 & 1.0690715004486243979 \\
2 & 1.0562222079392582146 \\
3 & 1.0551161172778792415 \\
4 & 1.0550572191430135037 \\
5 & 1.0550686438367760540 \\
6 & 1.0550735765579806638 \\
7 & 1.0550745305844060375 \\
8 & 1.0550745824286880798 \\
9 & 1.0550745442484434695 \\
10 & 1.0550745270114378657 \\
11 & 1.0550745231398621545 \\
12 & 1.0550745229039529066 \\
13 & 1.0550745231150647888 \\
14 & 1.0550745232236878646 \\
15 & 1.0550745232523526379 \\
16 & 1.0550745232552728533 \\
17 & 1.0550745232539308640 \\
18 & 1.0550745232530327777 \\
19 & 1.0550745232527427584 \\
20 & 1.0550745232526945061 \\
21 & 1.0550745232527008669 \\
22 & 1.0550745232527090401 \\
23 & 1.0550745232527124342 \\
24 & 1.0550745232527132611 \\
25 & 1.0550745232527133099 \\
26 & 1.0550745232527132427 \\
27 & 1.0550745232527132021 \\
28 & 1.0550745232527131884 \\
32 & 1.0550745232527131859 \\
30 & 1.0550745232527131861 \\
31 & 1.0550745232527131865 \\
& 1.0550745232527131867 \\
33 & 1.0550745232527131868 \\
\hline
\end{tabular}

For $\alpha=1 / 10$ and $\beta=1$, the equation for the inverse is given by

$$
-z=E_{1 / 10,1}^{-1}(w)=-\sum_{k=1}^{32} b_{k}(1-w)^{k}-\frac{1}{\Gamma(9 / 10)} \frac{(1-w)^{33}}{w}
$$

where $1 / \Gamma(9 / 10)=0.93577872091287277317 \ldots$ and the coefficients $b_{k}$ are given in Table 11 .

For $\alpha=1 / 3$ and $\beta=2$, the equation for the inverse is given by

$$
-z=E_{1 / 3,2}^{-1}(w)=-\sum_{k=1}^{91} b_{k}(1-w)^{k}-\frac{1}{\Gamma(5 / 3)} \frac{(1-w)^{92}}{w},
$$

where $1 / \Gamma(5 / 3)=1.1077321674324724694 \ldots$ and the coefficients $b_{k}$ are given in Table 12 . 
TABLE 17: Coefficients $b_{k}$ for the inverse Mittag-Leffler function $-z=$ $E_{1 / 8,2}^{-1}(w)$.

\begin{tabular}{lc}
\hline$k$ & $b_{k}$ \\
\hline 1 & 1.0594605373309141740 \\
2 & 1.0495986360361847141 \\
3 & 1.0488511439374145246 \\
4 & 1.0488179924913977120 \\
5 & 1.0488245771415334177 \\
6 & 1.0488269350920946859 \\
7 & 1.0488273133611761220 \\
8 & 1.0488273231954621562 \\
9 & 1.0488273085970012075 \\
10 & 1.0488273034622550713 \\
11 & 1.0488273025690472404 \\
12 & 1.0488273025660372711 \\
13 & 1.0488273026212509980 \\
14 & 1.0488273026418176097 \\
15 & 1.0488273026458036695 \\
16 & 1.0488273026458799051 \\
17 & 1.0488273026456156560 \\
18 & 1.0488273026455029277 \\
19 & 1.0488273026454771037 \\
20 & 1.0488273026454752946 \\
21 & 1.0488273026454766352 \\
22 & 1.0488273026454773654 \\
23 & 1.0488273026454775715 \\
24 & 1.0488273026454775998 \\
26 & 1.0488273026454775944 \\
28 & 1.0488273026454775895 \\
& 1.0488273026454775877 \\
& 1.0488273026454775873 \\
\hline &
\end{tabular}

For $\alpha=1 / 4$ and $\beta=2$, the equation for the inverse is given by

$$
-z=E_{1 / 4,2}^{-1}(w)=-\sum_{k=1}^{63} b_{k}(1-w)^{k}-\frac{1}{\Gamma(7 / 4)} \frac{(1-w)^{64}}{w},
$$

where $1 / \Gamma(7 / 4)=1.0880652521310173081 \ldots$ and the coefficients $b_{k}$ are given in Table 13 .

For $\alpha=1 / 5$ and $\beta=2$, the equation for the inverse is given by

$$
-z=E_{1 / 5,2}^{-1}(w)=-\sum_{k=1}^{46} b_{k}(1-w)^{k}-\frac{1}{\Gamma(9 / 5)} \frac{(1-w)^{47}}{w},
$$

where $1 / \Gamma(9 / 5)=1.0736712740308343279 \ldots$ and the coefficients $b_{k}$ are given in Table 14 .

For $\alpha=1 / 6$ and $\beta=2$, the equation for the inverse is given by

$$
-z=E_{1 / 6,2}^{-1}(w)=-\sum_{k=1}^{38} b_{k}(1-w)^{k}-\frac{1}{\Gamma(11 / 6)} \frac{(1-w)^{39}}{w},
$$

TABLE 18: Coefficients $b_{k}$ for the inverse Mittag-Leffler function $-z=$ $E_{1 / 9,2}^{-1}(w)$.

\begin{tabular}{lc}
\hline$k$ & $b_{k}$ \\
\hline 1 & 1.0521837208912933272 \\
2 & 1.0443758743852848005 \\
3 & 1.0438473880668843474 \\
4 & 1.0438274217379570018 \\
5 & 1.0438314036520292794 \\
6 & 1.0438326220325679883 \\
7 & 1.0438327879063812056 \\
8 & 1.0438327888679432382 \\
9 & 1.0438327828921939645 \\
10 & 1.0438327811719258151 \\
11 & 1.0438327809347620508 \\
12 & 1.0438327809451382984 \\
13 & 1.0438327809605757500 \\
14 & 1.0438327809650783542 \\
15 & 1.0438327809657296823 \\
16 & 1.0438327809656925712 \\
17 & 1.0438327809656403057 \\
18 & 1.0438327809656237760 \\
19 & 1.0438327809656210337 \\
20 & 1.0438327809656210870 \\
21 & 1.0438327809656212900 \\
22 & 1.0438327809656213646 \\
23 & 1.0438327809656213797 \\
24 & 1.0438327809656213804 \\
25 & 1.0438327809656213796 \\
26 & 1.0438327809656213792 \\
27 & 1.0438327809656213791 \\
\hline & \\
\hline &
\end{tabular}

where $1 / \Gamma(11 / 6)=1.0630880477938076525 \ldots$ and the coefficients $b_{k}$ are given in Table 15 .

For $\alpha=1 / 7$ and $\beta=2$, the equation for the inverse is given by

$$
-z=E_{1 / 7,2}^{-1}(w)=-\sum_{k=1}^{33} b_{k}(1-w)^{k}-\frac{1}{\Gamma(13 / 7)} \frac{(1-w)^{34}}{w},
$$

where $1 / \Gamma(13 / 7)=1.0550745232527131868 \ldots$ and the coefficients $b_{k}$ are given in Table 16 .

For $\alpha=1 / 8$ and $\beta=2$, the equation for the inverse is given by

$$
-z=E_{1 / 8,2}^{-1}(w)=-\sum_{k=1}^{28} b_{k}(1-w)^{k}-\frac{1}{\Gamma(15 / 8)} \frac{(1-w)^{29}}{w}
$$

where $1 / \Gamma(15 / 8)=1.0488273026454775873 \ldots$ and the coefficients $b_{k}$ are given in Table 17. 
TABLE 19: Coefficients $b_{k}$ for the inverse Mittag-Leffler function $-z=$ $E_{1 / 10,2}^{-1}(w)$.

\begin{tabular}{lc}
\hline$k$ & $b_{k}$ \\
\hline 1 & 1.0464858468535605019 \\
2 & 1.0401508480560282303 \\
3 & 1.0397635665515442126 \\
4 & 1.0397508768185132109 \\
5 & 1.0397533885835924940 \\
6 & 1.0397540594403559415 \\
7 & 1.0397541383662199550 \\
8 & 1.0397541376605313080 \\
9 & 1.0397541350429188308 \\
10 & 1.0397541344064541458 \\
11 & 1.0397541343357544813 \\
12 & 1.0397541343417232130 \\
13 & 1.0397541343464255068 \\
14 & 1.0397541343475460327 \\
15 & 1.0397541343476671587 \\
16 & 1.0397541343476507388 \\
17 & 1.0397541343476394778 \\
18 & 1.0397541343476366678 \\
19 & 1.0397541343476363331 \\
20 & 1.0397541343476363726 \\
21 & 1.0397541343476364046 \\
23 & 1.0397541343476364135 \\
24 & 1.0397541343476364148 \\
25 & 1.0397541343476364147 \\
& 1.0397541343476364146 \\
\hline
\end{tabular}

For $\alpha=1 / 9$ and $\beta=2$, the equation for the inverse is given by

$$
-z=E_{1 / 9,2}^{-1}(w)=-\sum_{k=1}^{27} b_{k}(1-w)^{k}-\frac{1}{\Gamma(17 / 9)} \frac{(1-w)^{28}}{w},
$$

where $1 / \Gamma(17 / 9)=1.0438327809656213791 \ldots$ and the coefficients $b_{k}$ are given in Table 18 .

For $\alpha=1 / 10$ and $\beta=2$, the equation for the inverse is given by

$$
-z=E_{1 / 10,2}^{-1}(w)=-\sum_{k=1}^{25} b_{k}(1-w)^{k}-\frac{1}{\Gamma(19 / 10)} \frac{(1-w)^{26}}{w},
$$

where $1 / \Gamma(19 / 10)=1.0397541343476364146 \ldots$ and the coefficients $b_{k}$ are given in Table 19.

\section{Summary}

A finite series representation of the inverse Mittag-Leffler function has been found for a range of the parameters $\alpha$ and $\beta$; specifically $0<\alpha<1 / 2$ for $\beta=1$ and for $\beta=2$. Various properties of the coefficients $b_{k}$ in the finite series have been examined. In addition, a formula for $b_{k}$ as $k \rightarrow \infty$ is established and the limiting cases were investigated. These properties are illustrated in 16 examples of inverse MittagLeffler functions. Determining the value of the argument of a Mittag-Leffler function given the value of the function is not an easy problem and the finite series representation of the inverse Mittag-Leffler function greatly expedites their evaluation and represents a significant advancement.

\section{Conflict of Interests}

The authors declare that there is no conflict of interests regarding the publication of this paper.

\section{References}

[1] A. Erdélyi, Ed., Higher Transcendental Functions, vol. 3, McGraw-Hill, New York, NY, USA, 1955.

[2] I. Podlubny, Fractional Differential Equations, vol. 198 of Mathematics in Science and Engineering, Academic Press, San Diego, Calif, USA, 1999.

[3] A. A. Kilbas, H. M. Srivastava, and J. J. Trujillo, Theory and Applications of Fractional Differential Equations, vol. 204 of North-Holland Mathematics Studies, Elsevier Science B.V., Amsterdam, The Netherlands, 2006.

[4] K. Diethelm, The Analysis of Fractional Differential Equations, vol. 2004 of Lecture Notes in Mathematics, Springer, New York, NY, USA, 2010.

[5] R. Hilfer, Applications of Fractional Calculus in Physics, World Scientific, Singapore, 2000.

[6] F. Mainardi, Fractional Calculus and Waves in Linear Viscoelasticity, Imperial College Press, London, UK, 2010.

[7] R. L. Magin, Fractional Calculus in Bioengineering, Begell House Publishers, Redding, Conn, USA, 2006.

[8] F. Mainardi and R. Gorenflo, "On Mittag-Leffler-type functions in fractional evolution processes," Journal of Computational and Applied Mathematics, vol. 118, no. 1-2, pp. 283-299, 2000.

[9] R. L. Magin, "Fractional calculus in bioengineering," Critical Reviews in Biomedical Engineering, vol. 32, no. 1, pp. 1-104, 2004.

[10] M. N. Berberan-Santos, "Properties of the Mittag-Leffler relaxation function," Journal of Mathematical Chemistry, vol. 38, no. 4, pp. 629-635, 2005.

[11] I. S. Gupta and L. Debnath, "Some properties of the MittagLeffler functions," Integral Transforms and Special Functions, vol. 18, no. 5-6, pp. 329-336, 2007.

[12] H. J. Haubold, A. M. Mathai, and R. K. Saxena, "MittagLeffler functions and their applications," Journal of Applied Mathematics, vol. 2011, Article ID 298628, 51 pages, 2011.

[13] R. Hilfer and H. J. Seybold, "Computation of the generalized Mittag-Leffler function and its inverse in the complex plane," Integral Transforms and Special Functions, vol. 17, no. 9, pp. 637$652,2006$.

[14] K. Knopp, Theory and Application of Infinite Series, Dover, New York, NY, USA, 1990.

[15] M. Abramowitz and I. A. Stegun, Handbook of Mathematical Functions with Formulas, Graphs, and Mathematical Tables, Dover, New York, NY, USA, 1965.

[16] H. B. Dwight, Tables of Integrals and Other mathematical Data, The Macmillan Company, New York, NY, USA, 4th edition, 1961. 
[17] W. H. Beyer, CRC Standard Mathematical Tables, CRC Press, Boca Raton, Fla, USA, 27th edition, 1984.

[18] P. Morse and H. Feshbach, Methods of Theoretical Physics, vol. 1, McGraw-Hill Book, New York, NY, USA, 1953.

[19] J. McMahon, "On the general term in the reversion of series," Bulletin of the American Mathematical Society, vol. 3, no. 7, pp. 170-172, 1894.

[20] E. P. Adams and R. L. Hippisley, Smithsonian Mathematical Formulae and Tables of Elliptical Functions, Smithsonian miscellaneous Collections 74(1), Publication 2672, Smithsonian Institution, Washington, DC, USA, 1939.

[21] J. Riordan, Combinatorial Identities, John Wiley \& Sons, New York, NY, USA, 1968.

[22] C. E. Van Orstrand, "Reversion of power series," Philosophical Magazine, vol. 19, series 6, pp. 366-376, 1910.

[23] J. W. Müller, "Some observations on the reversion of series," Rapport BIPM-85/1, Bureau International des Poids et Mesures, Sèvres, France, 1985.

[24] K. S. Miller and S. G. Samko, "A note on the complete monotonicity of the generalized Mittag-Leffler function," Real Analysis Exchange, vol. 23, no. 2, pp. 753-755, 1997.

[25] K. S. Miller and S. G. Samko, "Completely monotonic functions," Integral Transforms and Special Functions, vol. 12, no. 4, pp. 389-402, 2001 


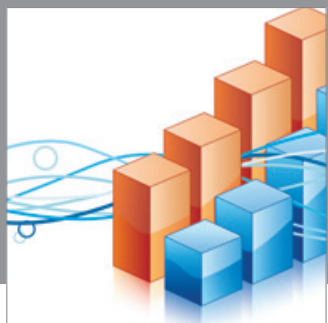

Advances in

Operations Research

mansans

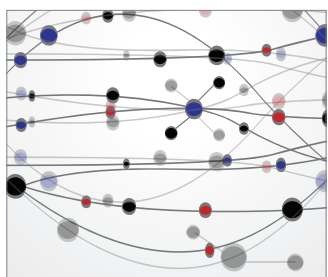

The Scientific World Journal
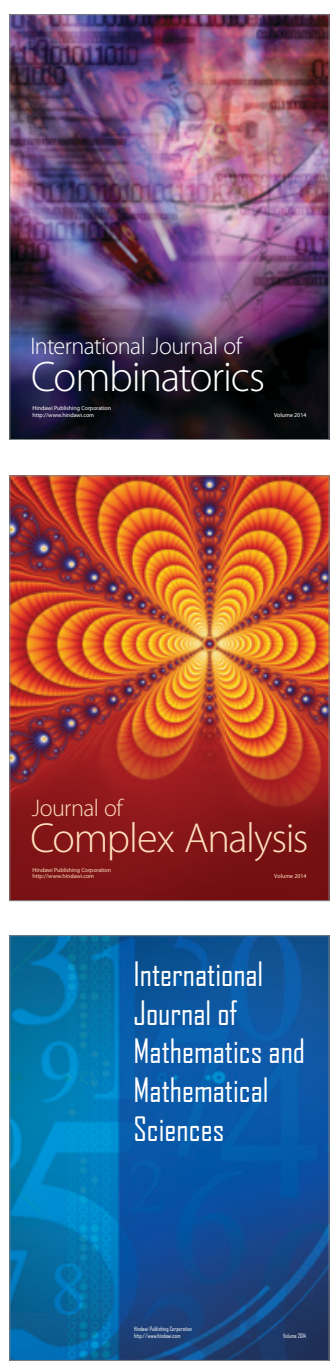
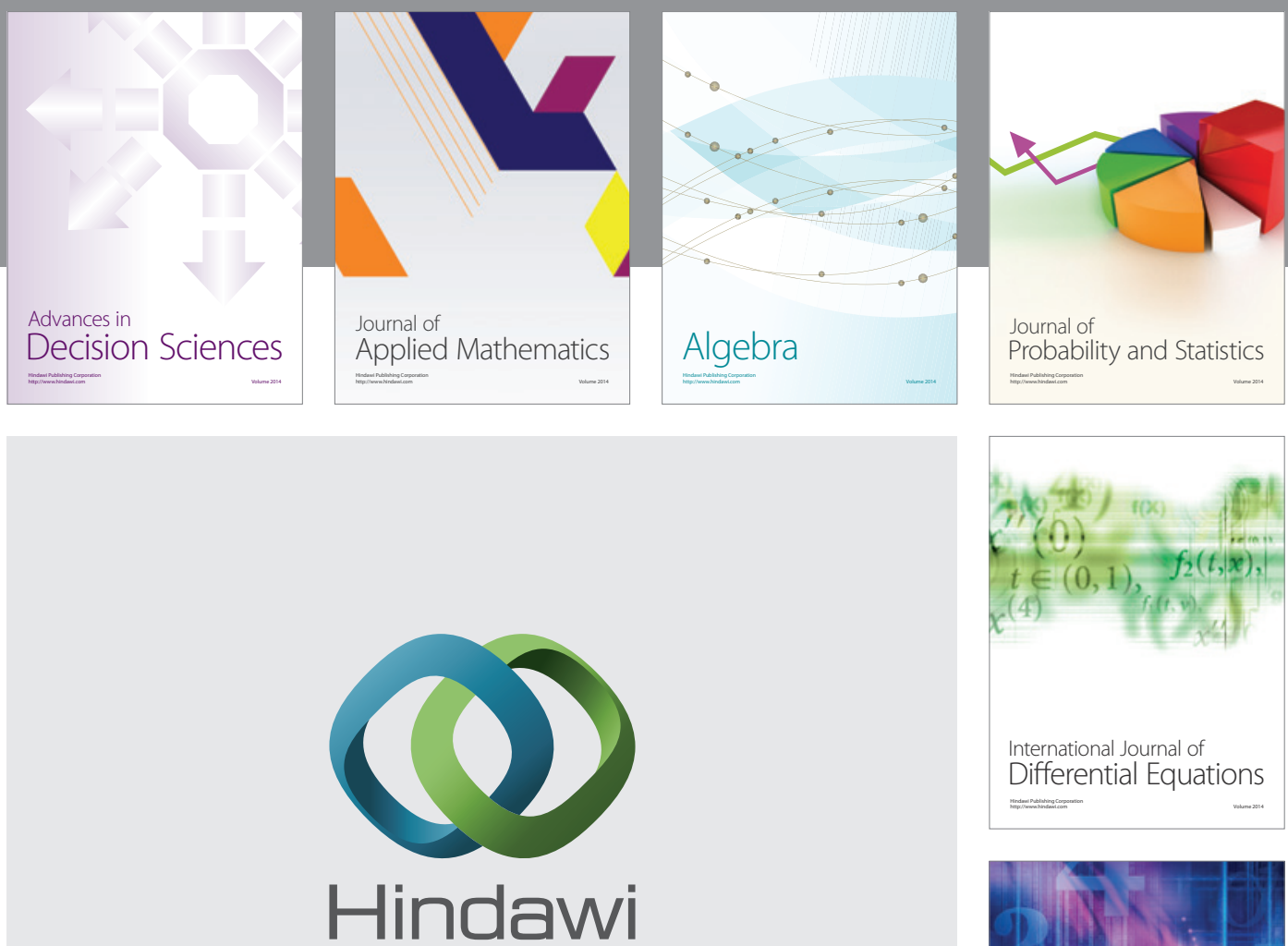

Submit your manuscripts at http://www.hindawi.com
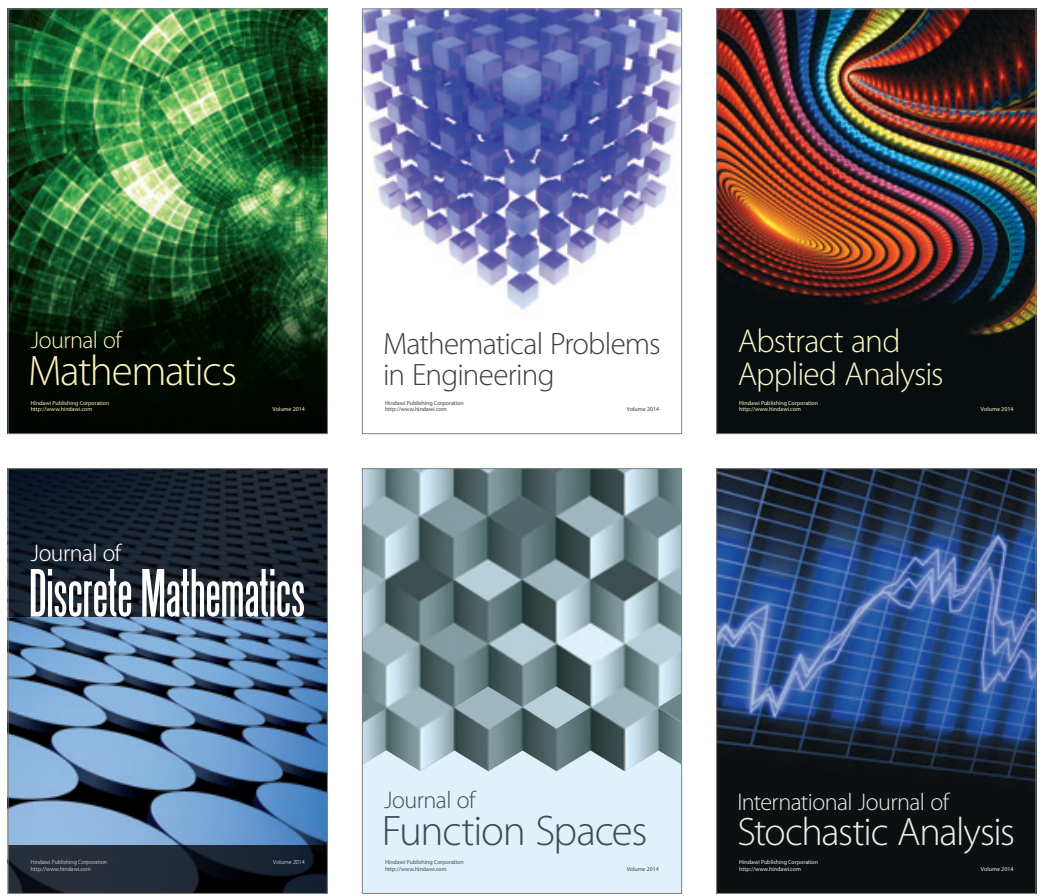

Journal of

Function Spaces

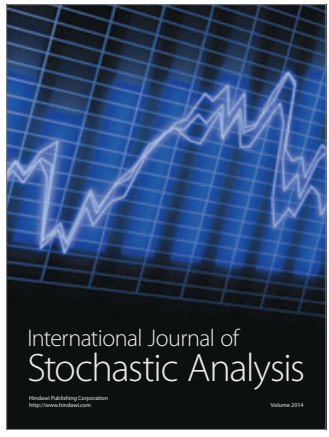

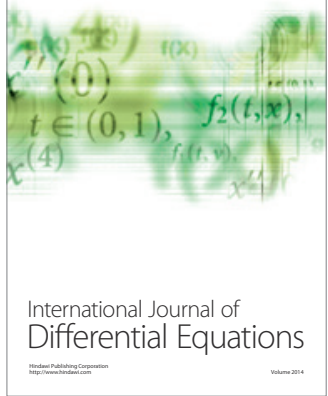
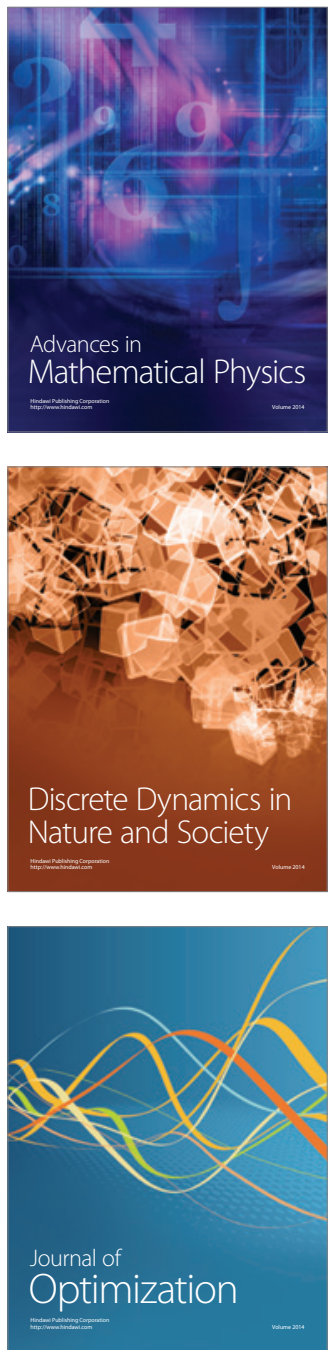